\title{
Unfolding the mechanical properties of buckypaper composites: nano- to macro-scale coupled atomistic-continuum simulations
}

\author{
Y. Chandra ${ }^{1} \cdot$ S. Adhikari ${ }^{2} \cdot$ S. Mukherjee ${ }^{1} \cdot$ T. Mukhopadhyay $^{3}$
}

Received: 4 August 2021 / Accepted: 14 October 2021 / Published online: 19 January 2022

(c) The Author(s) 2022

\begin{abstract}
Carbon-based nanostructures are receiving increasing attention over the past two decades due to their unprecedented multifunctional features. However, the macro-scale structural applications of these nanostructures have not yet come to full fruition due to the involvement of complex multi-scale computations and manufacturing. Recently, the research community has started investigating buckypaper, which can be described as a sheet or membrane developed using a network of bundles of singlewall carbon nanotubes, multi-wall carbon nanotubes, or a mixture of both. This article aims to focus on the computational bridging of different length scales involving six levels in the range of nano- to macro-scale behaviour concerning buckypaper composites. The sequential derivatives of carbon at six levels, as analyzed in this paper, involve graphene, CNT, CNT bundle, buckypaper, and buckypaper composite automotive components. Here, we adopt a coupled atomistic-continuum modelling approach for the multi-level simulations. Graphene, CNTs, and CNT bundles are modelled using atomistic simulations, while the buckypaper and its composites are modelled using equivalent beam representations for the bundles and continuum solid representation for resin. At the macro-scale, an industry-relevant multi-material composite automotive component has been investigated, wherein the buckypaper is proposed to be embedded involving sheet moulding compound and carbon prepreg. The current simulations have led to the determination of mechanical properties at each level of the carbon-based materials and their mutual dependence. The numerical results demonstrate that a buckypaper composite can enhance the natural frequency and stiffness up to 25 and $37 \%$ with respect to conventional monolithic metallic designs, while reducing the weight by $57 \%$. Such outcomes lead to the realization that carbon-based nanostructural derivative in the form of buckypaper can significantly improve the mechanical properties of advanced lightweight structural components as reinforcements for the next generation of aerospace and automotive structures.
\end{abstract}

S. Adhikari

Sondipon.Adhikari@glasgow.ac.uk

T. Mukhopadhyay

tanmoy@ iitk.ac.in

1 College of Engineering, Swansea University, Swansea, UK

2 James Watt School of Engineering, University of Glasgow, Glasgow, UK

3 Department of Aerospace Engineering, Indian Institute of Technology Kanpur, Kanpur, India 


\section{Graphical abstract}

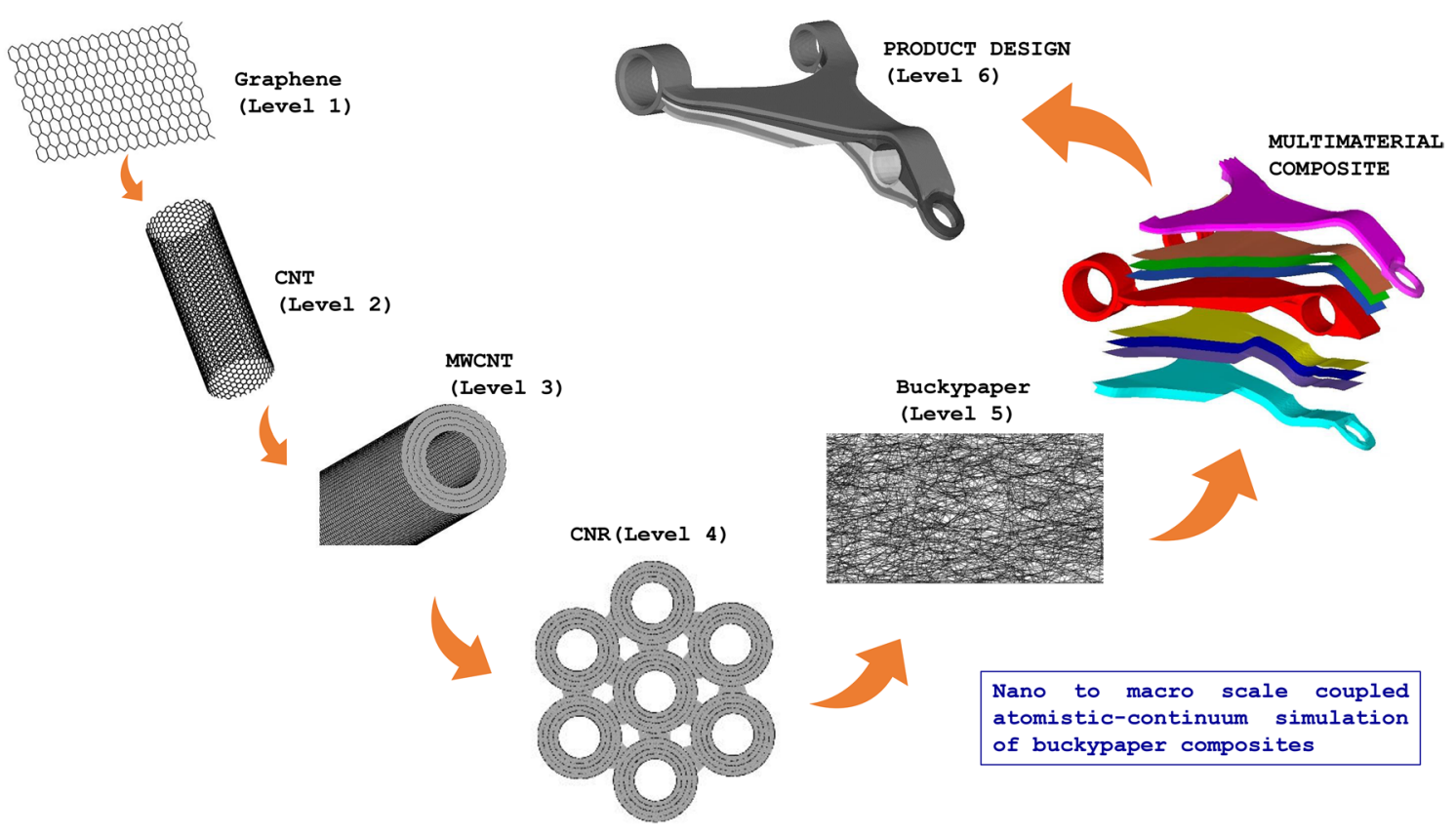

Keywords Graphene and its derivatives $\cdot \mathrm{CNT}$ and CNT bundles $\cdot$ Atomistic-continuum modelling $\cdot$ Buckypaper composites · Automotive light-weighting

\section{Introduction}

Graphene and its associated nanostructures (GANS) are considered as miracle materials of the future due to their unprecedented mechanical and other multi-functional properties $[22,23,63,102,113,116,141]$. However, the application of these materials seems to be largely limited to nanotechnology due to computational and fabrication hurdles at a higher length scale. Currently, networked paper-like formats of these nanomaterials are gaining increasing attention for micro- and macro-scale applications. The success of such investigations could result in an ultra-lightweight revolution in the mainstream engineering industries such as aerospace and automobile. The properties of paper formats of graphene and carbon nanotubes (PFGCN) are studied with the aid of experimental and numerical methodologies. Since the GANS are the building blocks of PFGCN materials, the mechanical properties of PFGCN are strongly dependent on the physical and mechanical properties of the underlying GANS. Thus, the numerical simulation of PFGCN will necessitate the numerical simulation of GANS at multiple levels. GANS's numerical simulation involves a high-fidelity representation of GANS molecules, which means that the atoms and their interactions need to be considered in the simulations. The representative volume element approximations (RVE) offer reasonable accuracy in determining the mechanical properties of these nanostructures [21, 24-29]. On the other hand, the PFGCN structures can be represented by low-fidelity finite-element models considering the equivalent properties of GANS without the loss of accuracy [157, 171]. Thus, the entire simulation involves an atomistic-continuum multi-level numerical framework starting from the atomic bond properties to the network of PFGCN embedded in matrix materials for manufacturing effective macro-scale structural components. We aim to characterize the effective mechanical properties at each level and bridging different length scales for seamless propagation of the nanolevel properties to the macro-scale structure level.

Buckypaper can be described as a sheet or membrane developed using a network of bundles of single-wall carbon nanotubes (SWCNT) or multi-wall carbon nanotubes (MWCNT). The bundles can also be a mix of SWCNTs and MWCNTs to attain a mix of desired properties [37, 64]. Rinzler et al. [140] prepared a batch of SWCNT using dual pulsed laser vaporization process and then purified the resulting material using nitric acid. This process resulted in a free-standing mat of SWCNT, which the researchers termed as "buckypaper". High mechanical performance composites of buckypapers have been successfully synthesized using a prepreg approach $[34,35]$. In general, it is a well-known fact that the CNTs (carbon nanotubes) offer exceptional mechanical properties $[9,43,110,134,160]$. However, it 
is nearly impossible to synthesize high-performance polymeric composites with carbon nanotubes (CNT) as primary fillers or fibres due to dispersion and alignment issues. Typically, the attainable and feasible weight fraction of CNTs in the nanocomposites is less than 5\% [19, 21, 130, 161]. To realize higher weight fractions of CNTs in composites, researchers resorted to CNT networks in the form of buckypaper. If many CNTs are dispersed in an aqueous solution, the individual CNTs attract each other and form a thin film when the solution is filtered out. Such a thin film of CNTs is termed buckypaper. The buckypaper is also referred to as "free-standing CNT paper" or "film of randomly arranged bundles of CNTs". The chopped arrangements of CNT particles within the buckypaper lead to porosity. The porous sheet of buckypaper has been claimed to be stronger and lighter than typical steel grades $[34,35]$. Unlike graphene and CNT, the buckypaper membrane can be macroscopic in nature and is easy to handle. The samples of buckypapers of thickness ranging from $\mu \mathrm{ms}$ to $\mathrm{mm}$ are commercially synthesized. The orientation of CNTs in the buckypaper can be controlled by a magnetic field and/or a compressive load during the stage of synthesis. The presence of pure CNTs at appropriate orientations and tight entanglements between CNTs can make buckypapers an order of magnitude stronger, lighter, and stiffer than conventional engineering materials such as steel alloys, aluminium alloys, titanium alloys, glass fibres, aramid fibres, and carbon fibres. Therefore, buckypaper has a tremendous potential to act as the primary reinforcing material in modern reinforced plastics and structural composites.

In the last 2 decades, a large volume of research work has taken place to develop the nanoscale science and engineering of GANS [71, 123]. The mechanical properties of individual graphene sheets and CNTs have been widely studied [87, 137, $158,167]$ including their composites [29, 132]. These studies have also progressed to bilayered [22, 23] and multilayered systems $[73,111]$. Compared to the research works concerning a single layer, bilayer, and multilayer systems, that of the bundled formats is found to be significantly less [39, 92]. Even a lesser number of research investigations on PFGCN materials at nanoscale can be traced in the literature [38, 184]. The PFGCN materials have been successfully produced in dimensions up to several meters $[159,177]$. However, the literature pertaining to the mechanical properties of such meter-scale PFGCN materials is very scarce. Therefore, there exists a strong rationale to investigate PFGCN materials at larger dimensions along with scalable production, which is essential to bring graphene-based materials closer to the demands of structural industries [180]. The crucial aspects of multiscale simulations and scale-bridging ranging from graphene (nanoscale) to engineering products involving buckypaper composites (macro-scale) have not been investigated thoroughly. It can be noted in this context that different forms of structural composites are being increasingly utilized for their high specific strength and stiffness along with other attractive mechanical features $[41,63,66,74,80-82,117,119,149,150$, 169]. PFGCN-based composites could further improve the multi-functional mechanical performances of such composites.

This paper aims to propose a seamlessly coupled atomistic-continuum simulation for characterizing the mechanical properties of buckypaper by performing simulations at six distinct levels and propagating the equivalent properties hierarchically in the order of graphene, CNT, MWCNT, CNT bundle, buckypaper, and buckypaper composites along with its practical applications as engineering components (refer to Figs. 1 and 4). Hereafter, this paper is organised as follows. The multi-level simulation framework covering nano- to macro-scale is provided systematically in Sect. 2 . The simulation methodology for modelling graphene at the atomic level is presented in Sect. 2.1. This involves finding the mechanical equivalence between covalent bond stiffness and inter-atomic potentials. A similar methodology has also been adopted to model SWCNT and is explained in Sect. 2.2. The atomistic simulation methodology has been extended from SWCNT to MWCNT in Sect. 2.3. This section also deals with inter-wall interactions in an MWCNT. Furthermore, the MWCNT bundles that form the backbone of buckypapers have been modelled at the nanoscale as described in Sect. 2.4. Such MWCNT bundles are also referred to as carbon nanoropes (CNR) in the scientific community $[15,16]$. A customised algorithm has been developed here to distribute nanoropes in a buckypaper randomly. Both wavy and aligned nanoropes have been considered in the buckypaper. The detailed algorithm and a strategy to model buckypaper composite have been explained in Sect. 2.5. At the next stage, the derived buckypaper composite properties have been used to simulate a critical automotive suspension part (control arm), as discussed in Sect. 2.6. The structure-level mechanical performance of the control arm has been assessed using static and free vibration analyses here. The numerical results concerning equivalent mechanical properties at the above-mentioned six levels have been discussed in Sect. 3, covering Young's modulus, shear modulus and Poisson's ratio of graphene, SWCNT, MWCNT, MWCNT bundle, and buckypaper composite. The numerical results of buckypaper composites have been utilized as one of the primary input properties to assess the static and vibrational performances (involving deflection, mode shapes, and natural frequencies) of the control arm, as presented in Sect. 3.6. Finally, Sect. 4 provides the concluding remarks and a critical perspective on the potential impact of this paper. 


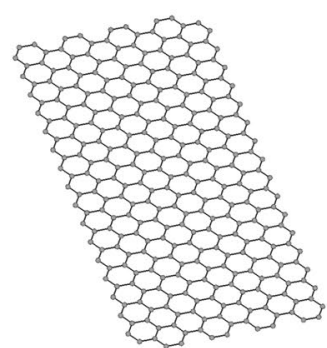

(a) Graphene

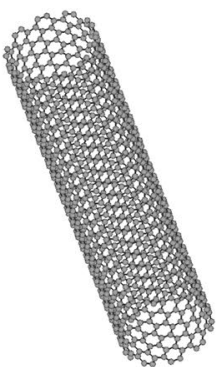

(b) SWCNT

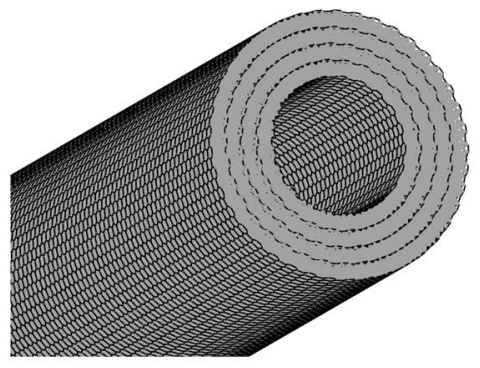

(c) MWCNT

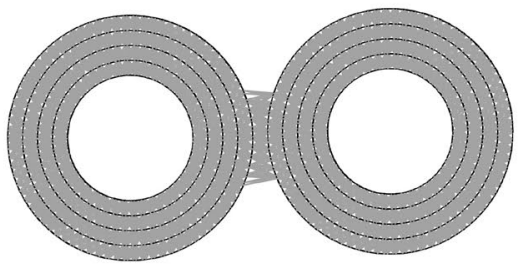

(d) Bundle of two MWCNTs

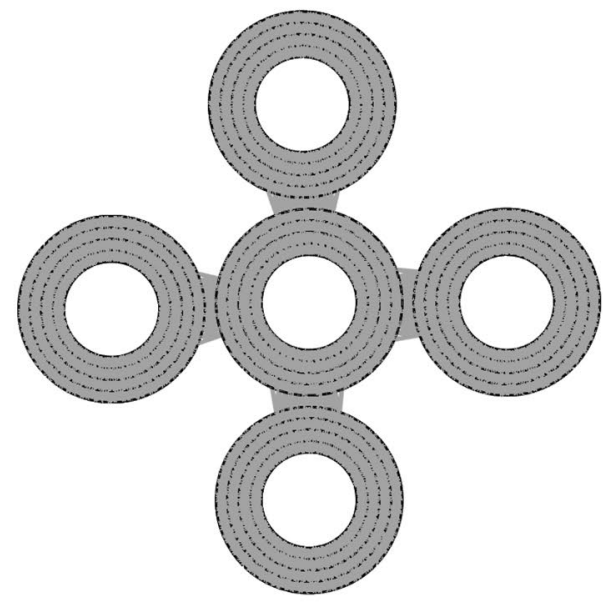

(f) Bundle of five MWCNTs

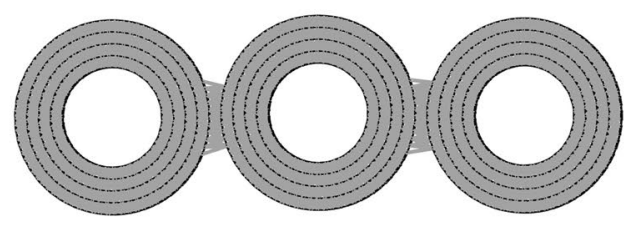

(e) Bundle of three MWCNTs

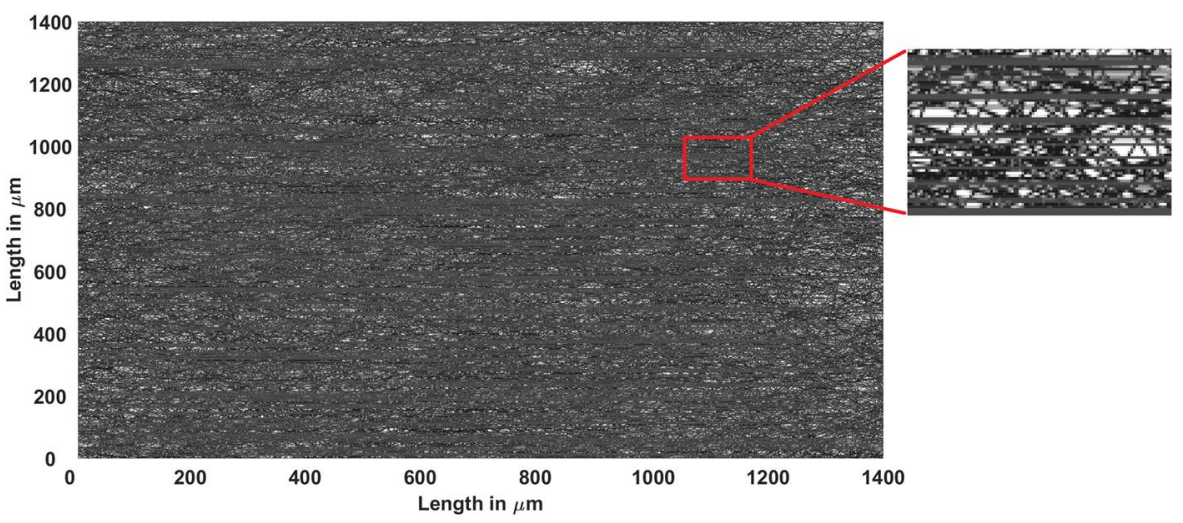

(h) A buckypaper with $40 \%$ stretched nanoropes.

Fig. 1 Typical representation of multi-level nanostructures investigated in the current study starting from graphene to MWCNT bundles and buckypaper. Composites made of such buckypapers as reinforcement are further used as macro-scale structural components 


\section{Multi-level simulation methodology covering nano- to macro-scales}

In this section, we provide the simulation framework for evaluating the effective elastic properties of graphene, CNT, MWCNT, CNT bundle, buckypaper, and buckypaper composites systematically. We start with the atomic bond-level properties and subsequently use a mechanical equivalence of such bonds to evaluate the effective elastic properties based on continuum-based finite-element approaches.

\subsection{Level 1: Atomistic-continuum simulation of graphene}

At this level of simulations, the mechanical equivalence of atomic bonds is used to model graphene sheets. The total inter-atomic potential energy can be derived as the sum of three individual energy terms relevant to bonding and nonbonding interactions [31]. Total strain energy $\left(E_{\mathrm{E}}\right)$ can be represented as the sum of energy contributions from bending of bonds $\left(E_{\mathrm{b}}\right)$, bond stretching $\left(E_{\mathrm{s}}\right)$, torsion of bonds $\left(E_{\mathrm{t}}\right)$ and nonbonded energies $\left(E_{\mathrm{nb}}\right)$ including the van der Waals interactions, the Coulombic energy, and the core repulsion

$E_{\mathrm{E}}=E_{\mathrm{s}}+E_{\mathrm{b}}+E_{\mathrm{t}}+E_{\mathrm{nb}}$.

The influence of bending and stretching is significant in case of small deformations as compared to all other energy components $[52,154]$. The deformation mechanisms for the multiplanar nanostructure are shown in Fig. $2 b-d$. The out-of-plane angular component becomes zero for monoplanar nanostructures such as graphene [114]. The total interatomic potential energy $\left(E_{\mathrm{E}}\right)$ can be represented as

$$
\begin{aligned}
E_{\mathrm{E}} & =E_{\mathrm{s}}+E_{\mathrm{bI}}+E_{\mathrm{bO}} \\
& =\frac{1}{2} k_{r}(\Delta l)^{2}+\frac{1}{2} k_{\theta}(\Delta \theta)^{2}+\frac{1}{2} k_{\theta}(\Delta \alpha)^{2},
\end{aligned}
$$

where $\Delta l, \Delta \theta$, and $\Delta \alpha$ denote the change in bond length, change in in-plane angle, and change in out-of-plane angle, respectively, as shown in Fig. 2. The quantities $k_{r}$ and $k_{\theta}$ are the force constants associated with bond stretching and
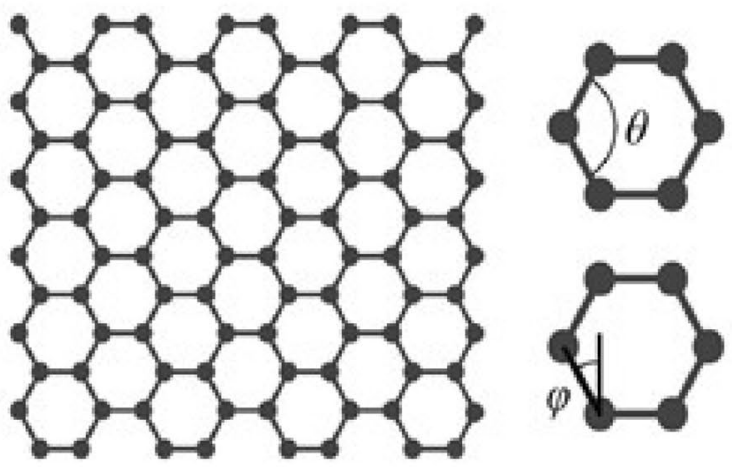

(a)

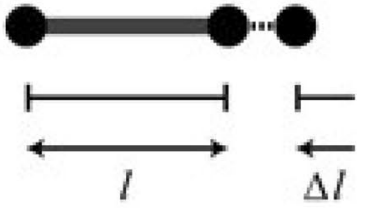

(b)

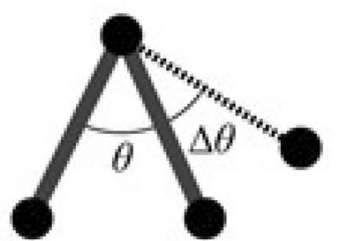

(c)

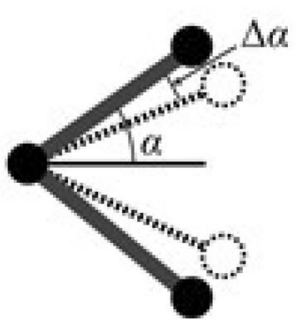

(d)

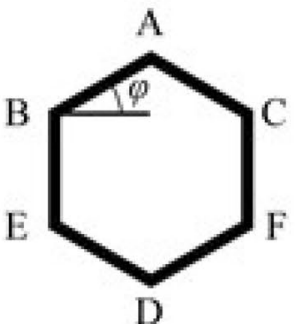

(e)

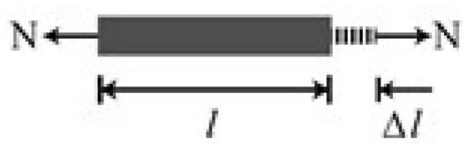

(f)

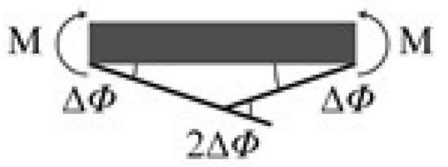

$(\mathrm{g})$
Fig. 2 Atomic-level mechanics of GANS. a Different views of hexagonal graphene nanostructure along with a representative unit cell. b Bond stretching induced strain energy. c In-plane angle variation induced strain energy. d Out-of-plane angle variation induced strain energy. e A hexagonal unit cell involving six idealized beam elements. f A beam element under the influence of pure tension. $\mathbf{g}$ A beam element under the influence pure bending 
bond bending, respectively. The first term in Eq. 2 represents strain energy relevant to stretching $\left(E_{\mathrm{s}}\right)$, while the other terms represent the strain energies due to in-plane $\left(E_{\mathrm{bI}}\right)$ and out-of-plane $\left(E_{\mathrm{bO}}\right)$ angle variations, respectively. The out-ofplane components are irrelevant in case of monolayer nanostructures (these are presented in the current paper for the sake of generality). Neglecting the out-of-plane components in monoplanar nanostructures like graphene, we get

$E_{\mathrm{E}}=\frac{1}{2} k_{r}(\Delta l)^{2}+\frac{1}{2} k_{\theta}(\Delta \theta)^{2}$.

The force constants of the atomic bonds $\left(k_{r}\right.$ and $\left.k_{\theta}\right)$ can be expressed in the form of structural equivalence [88]. From a structure-mechanical point of view (refer to Fig. 2e, f), the strain energy of a beam with Young's modulus $E$, crosssectional area $A$, length $l$, and moment of inertia $I$, under the influence of a pure axial force $N$ can be represented by

$U_{a}=\frac{1}{2} \int_{0}^{L} \frac{N^{2}}{E A} \mathrm{~d} l=\frac{1}{2} \frac{N^{2} l}{E A}=\frac{1}{2} \frac{E A}{l}(\Delta l)^{2}$.

The strain energies due to pure bending moment $M$ (refer to Fig. $2 \mathrm{~g}$ ) can be written as

$U_{b}=\frac{1}{2} \int_{0}^{L} \frac{M^{2}}{E I} \mathrm{~d} l=\frac{1}{2} \frac{E I}{l}(2 \Delta \phi)^{2}$.

Comparing Eq. 4 with the expression for strain energy due to stretching $\left(E_{\mathrm{s}}\right)$ (refer to Eq. 3), it can be concluded that $k_{r}=\frac{E A}{l}$. For bending, it is reasonable to assume that $2 \Delta \phi$ is equivalent to $\Delta \theta$ for in-plane angle variation (refer to Fig. 2g). Thus comparing Eq. 5 with the expression for strain energy due to in-plane ( $\left.E_{\mathrm{bI}}\right)$ angle variation (refer to Eq. 3), the following relation can be obtained: $k_{\theta}=\frac{E I}{l}$. It shows that there exists a mechanical equivalence between molecular mechanics parameters $\left(k_{r}\right.$ and $\left.k_{\theta}\right)$ and structural mechanics parameters ( $E A$ and $E I)$. Such mechanical equivalence can be used to derive equivalent beam (covalent bond) properties used in further atomistic simulations of graphene, which can essentially be idealized as a hexagonal network of beams. In the current work, the effective elastic moduli of the nanostructures are computed using equivalent beams representing covalent bonds.

After establishing the beam-level mechanical equivalence of atomic bonds, here, we describe the finite-element-based continuum-level framework for analyzing the network of such beams. For the atomistic-continuum simulations, the finite-element analysis code OPTISTRUCT [3] has been used, leading to characterization of the elastic behaviour of nanostructures. The covalent bonds are represented by 3D finite-element beams (element type: CBEAM), and the atoms are represented by finite-element nodes. The cross-sectional diameter and Young's modulus $(E)$ of the beam elements are computed using the equations of forcefield constants $k_{r}$ (stretching) and $k_{\theta}$ (torsional), as discussed in the preceding paragraphs. The numerical values of force field constants $k_{r}$ and $k_{\theta}$ are shown in Table 1 [114]. By substituting these values in the equations $k_{r}=\frac{E A}{l}$ and $k_{\theta}=\frac{E I}{l}$, essential parameters to model covalent bonds such as beam diameter $d$ and beam Young's modulus $E$ can be calculated. The bond lengths, which are essentially beam lengths, shown in Table 1 are obtained from the literature [114]. In the current work, the mechanical properties such as Young's modulus $(E)$, Poisson's ratio ( $v)$, and shear modulus $(G)$ have been computed using the atomistic FE models of the single-layer graphene sheet (SLGS) following the standard definition of the elastic moduli. The computed mechanical properties of SLGS are discussed in Sect. 3.1.

\subsection{Level 2: Atomistic-continuum simulation of SWCNT}

A single-wall carbon nanotube (SWCNT) is a cylindrical version of the graphene sheet. The atomistic model of SWCNT is shown in Fig. 1b. Here, the carbon atoms and their inter-atomic bonds have been generated using a similar atomistic computational methodology as developed for single-layer graphene (refer to Sect. 2.1). In the current work, the mechanical properties such as Young's modulus $(E)$, Poisson's ratio $(v)$, and shear modulus $(G)$ have been computed using the atomistic FE models of the SWCNT. For the atomistic-continuum simulations, the finite-element analysis code OPTISTRUCT [3] has been used, leading to characterization of the elastic behaviour. The covalent bonds are represented by 3D finite-element beams (element type: CBEAM), and the atoms are represented by finite-element nodes. The cross-sectional diameter and Young's modulus $(E)$ of the beam elements are computed using the equations of force-field constants $k_{r}$ (stretching) and $k_{\theta}$ (torsional). The numerical values of force-field constants $k_{r}$ and $k_{\theta}$ are shown in Table 1 [114]. By substituting these values in the equations $k_{r}=\frac{E A}{l}$ and $k_{\theta}=\frac{E I}{l}$, essential parameters to model covalent bonds such as beam diameter $d$ and beam Young's modulus $E$ can be calculated, as presented in Table 1. The bond lengths, which are essentially beam lengths, shown in

Table 1 Bond properties for carbon-carbon atomic interaction in graphene [114]

\begin{tabular}{|c|c|c|c|}
\hline$k_{r}$ in $\mathrm{N} \mathrm{nm}^{-1}$ & $k_{\theta}$ in $\mathrm{N} \mathrm{nm} \mathrm{rad}-2$ & $L$ in $\mathrm{nm}$ & $d$ in $\mathrm{nm}$ \\
\hline $6.52 \mathrm{e}-7$ & $8.76 \mathrm{e}-10$ & 0.142 & 0.146 \\
\hline
\end{tabular}


Table 1 are obtained from the literature [114]. The computed mechanical properties of SWCNTs are presented in Sect. 3.2.

\subsection{Level 3: Atomistic-continuum simulation of MWCNT}

In the current work, up to five walls have been considered in the mechanical analysis of MWCNT. The carbon atoms and their inter-atomic bonds have been generated using a similar atomistic computational methodology as developed for single-layer graphene (refer to Sect. 2.1). The wall-towall interactions in the MWCNTs need to be captured in the atomistic simulations. This can be implemented using the $\mathrm{L}-\mathrm{J}$ potential idealized as equivalent springs [115]. The equivalent axial force for an $\mathrm{L}-\mathrm{J}$ potential between pair of atoms $(i, j)$ belonging to different nanosheets can be defined as [145]

$F_{i j}=\frac{\partial V_{i j}}{\partial r}$,

where $r$ is the atomic displacement along $i j$ (layer-layer length). As per Girifalco et al. [60], the force between the atoms $(i, j)$ can also be represented by

$F_{i j}=-12 \epsilon\left[\left(\frac{r_{\min }}{y}\right)^{13}-\left(\frac{r_{\min }}{y}\right)^{7}\right]$,

where $y=r_{\min }+\delta r$, and $\delta r$ is the atomic displacement along the length ij. The $r_{\text {min }}$ (in $\AA$ ) is given by $2^{\frac{1}{6}} \sigma$, where $\sigma=(A / B)^{1 / 6}$. The quantities $B$ and $A$ are attractive and repulsive constants, respectively. The values of $B$ and $A$ are $24.3 \times 10^{3} \mathrm{eV}^{12}$ and $15.4 \times 10^{3} \mathrm{eV} \AA^{6}$, respectively [145]. In the atomistic FE models, spring elements form a non-linear connection between two adjacent layers of the multilayer structure represent $\mathrm{L}-\mathrm{J}$ interactions. The force-deflection curve for L-J springs has been calculated using the relation in Eq. 7. Within the finite-element analysis code OPTISTRUCT [3], the L-J springs of interlayer interactions are modelled by element type CBUSH and using the constitutive relation of Eq. 7 as input properties. An MWCNT with five walls and associated van der Waals springs is shown in Fig. 1c. The computed mechanical properties of MWCNTs are presented in Sect. 3.3.

\subsection{Level 4: Atomistic-continuum simulation of MWCNT bundles}

Analogous to carbon fibres produced by intermingling carbon filaments, it is also viable to produce nanoropes by intermingling CNTs. Such an arrangement can be formed by bundling SWCNTs or MWCNTs or a mix of both (refer to Fig. 1d-g). These bundles have been successfully used in various applications, including composite materials [93] and electronic displays [85]. In the current work, it has been assumed that the MWCNT of five walls forms the fundamental element of bundles. This assumption is based on the MWCNTs found in high-performance buckypapers [34, 35]. Four types of bundles have been trialled with two, three, five, and seven MWCNTs. Here, the tubes' arrangement in the seven MWCNT configuration is similar to the ones used in buckypapers presented by [1, 13]. Liu et al. [92] also modelled similar seven tube arrangements using a hybrid atomistic methodology. In this study, the carbon atoms and their inter-atomic bonds have been generated using a similar atomistic computational methodology as developed for single-layer graphene (refer to Sect. 2.1). The van der Walls interactions between the walls of individual MWCNTs have been modelled as described in Sect. 2.3. Van der Walls interactions have also been considered between the pairs of MWCNTs in the bundle. This is based on the $0.85 \mathrm{~nm}$ cut-off distance, beyond which the interaction would be negligible [152]. In the bundles' atomistic FE models, spring elements form a non-linear connection between a pair of MWCNTs, represent the L-J interactions. The force-deflection curve for L-J springs has been calculated by using the relation in Eq. 7. Within the finite-element analysis code OPTISTRUCT [3], the L-J springs of interlayer interactions are modelled by element type CBUSH and by using the constitutive curve obtained from Eq. 7 as input properties. Four variants of MWCNT bundles are shown in Fig. 1. The computed mechanical properties of MWCNT bundles are presented in Sect. 3.4.

\subsection{Level 5: Atomistic-continuum simulation of buckypaper composite}

To model buckypaper, the fibres of MWCNT bundles are represented as equivalent beams. The concept of equivalent beams in the context of nanotubes has been historically validated [44, 125, 144, 168]. The mechanical properties of MWCNT bundles (such as Young's modulus, shear modulus, and Poisson's ratio) computed in Sect. 3.4 have been used to define the equivalent beams here. A network of high aspect ratio beams forms the buckypaper, wherein each beam represents an MWCNT bundle (refer to Fig. 1h). It is assumed that each bundle is composed of seven MWCNTS, wherein each MWCNT is a five-walled CNT. The assumed arrangement of seven MWCNTs in a bundle is shown in Fig. $1 \mathrm{~g}[1,13,75]$. Two configurations of beams have been trialled in the current work, namely stretched beams and wavy beams. Stretched beam configurations are aligned and straight in nature, whereas wavy beam configurations display curvatures. The consideration of such beam configurations mimics the concept of stretch ratio presented by Cheng et al. [34, 35], wherein they demonstrated a high 


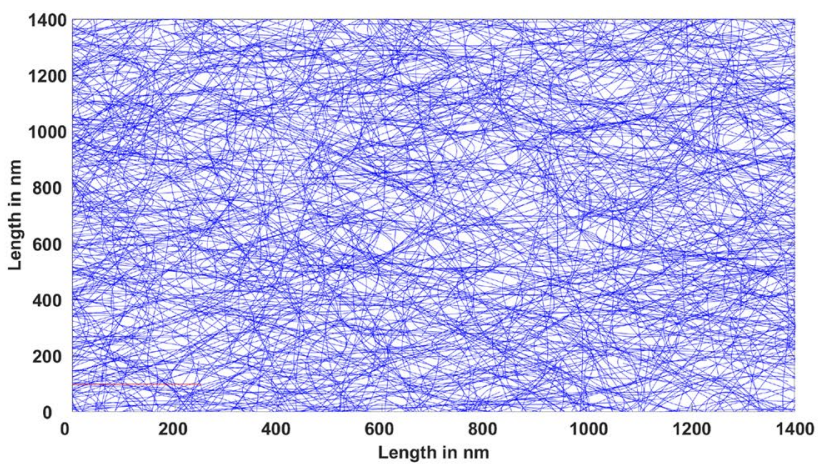

(a)

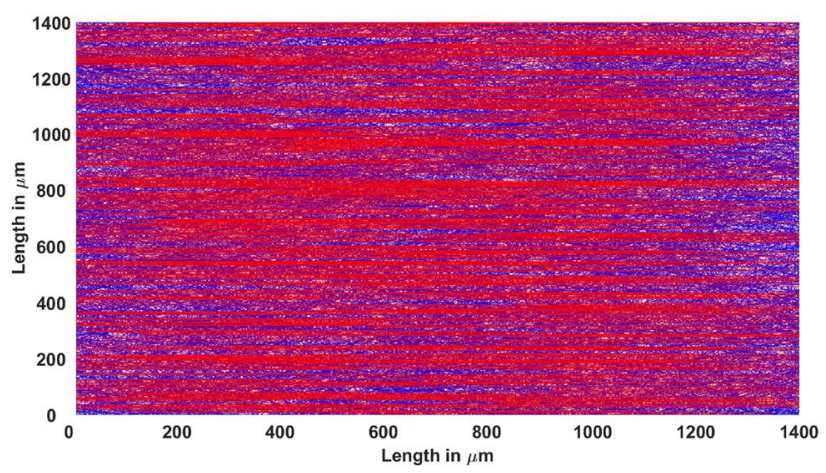

(c)

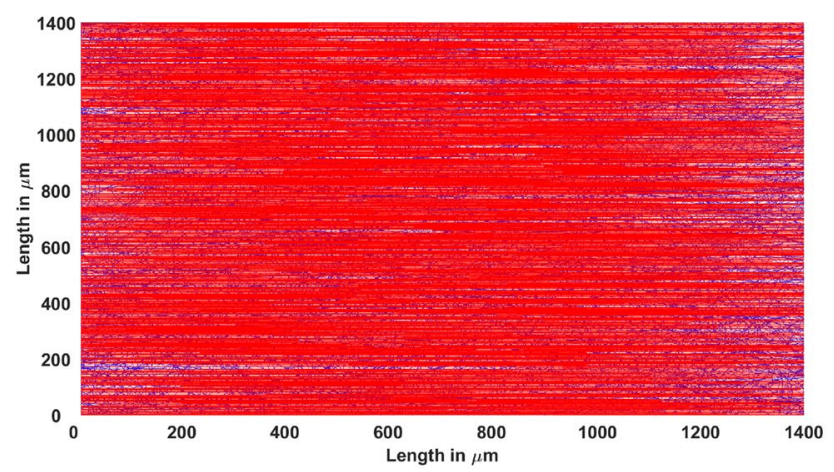

(e)

Fig. 3 Five variants of buckypaper and a mesh of the composite structure. a A buckypaper with 0\% stretched and 100\% wavy (unstretched) nanoropes. b A buckypaper with $20 \%$ stretched nanoropes. The rest of the nanoropes are wavy (un-stretched). c A buckypaper with $40 \%$ stretched nanoropes. The rest of the nanoropes are wavy (un-stretched). d A buckypaper with $60 \%$ stretched nanoropes. The

mechanical performance of buckypaper composites with the aid of high stretch ratio MWCNT bundles. The stretching of beams can be attained by either a mechanical force [34, 35] or by a magnetic field [61]. To demonstrate the influence of stretched beams on the mechanical performance of the buckypaper composite, five configurations have been modelled here. These configurations are based on the ratio

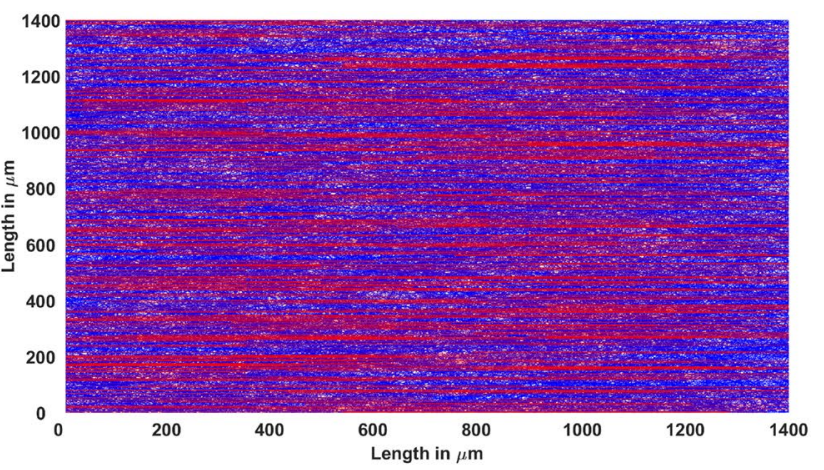

(b)

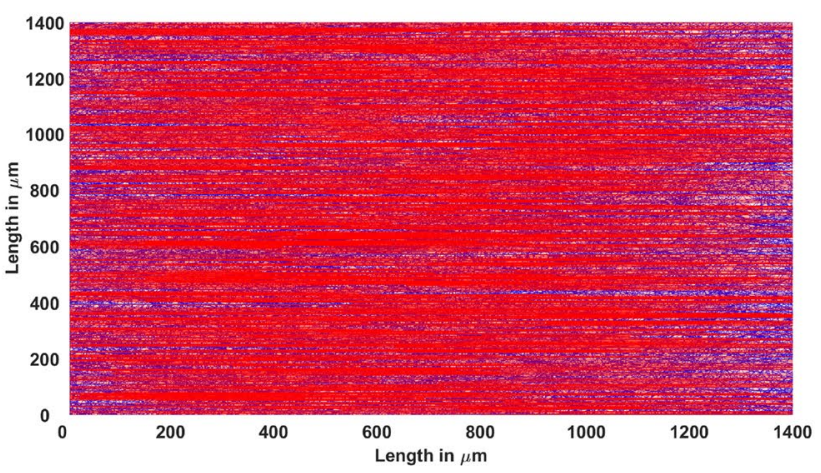

(d)

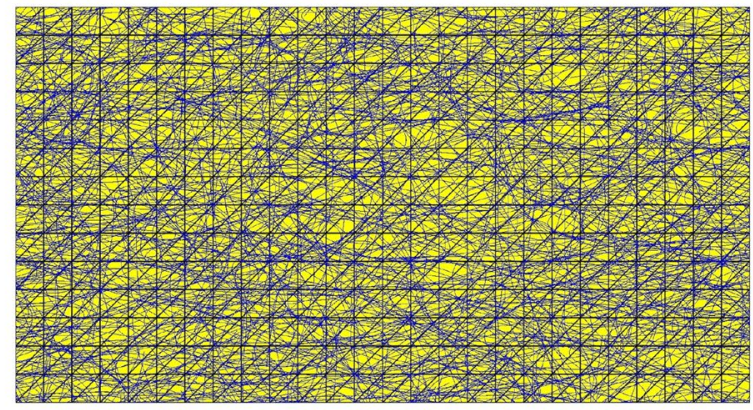

(f)

rest of the nanoropes are wavy (un-stretched). e A buckypaper with $80 \%$ stretched nanoropes. The rest of the nanoropes are wavy (unstretched). f A typical cross-sectional view of the meshed finite-element model of buckypaper composite. The image is taken with low density of nanoropes for the sake of visual clarity

between stretched to wavy (un-stretched) beams, as 0, 20, 40,60 , and $80 \%$ of stretched beams (refer to Fig. 3a-e, respectively). In these images, wavy beams are represented in blue colour and the stretched beams are represented in red colour. In a buckypaper, the MWCNT beams intersect with each other in various formats, which include entangled crossings, $Y$ and $T$ joints, nanowelding, nanosoldering, and 
joints of coated tubes. In the case of entangled crossings, the force transfer between beams occurs as a result of frictional contact and also due to weak van der Waals interactions [50, 120, 178]. The $Y$ and $T$ joints are formed when one end of a beam connects to other beam(s) resembling a $Y$ or $T$ shape $[84,106,186]$. Nanowelding is specific to irradiation, wherein an intersection between adjacent tubes leads to a defect and then resulting in bond formation $[120,163]$. Nanosoldering occurs when amorphous carbon deposits within the intersecting region of a pair of tubes [12, 120]. Joints of coated tubes are formed due to the depositions of coating materials at the intersections of tubes [12]. Berhan et al. [16] postulated that the above-mentioned formats of tube intersections could be represented by torsional springs. The stiffness of such torsional springs can be calculated by

$K=\frac{\bar{K} E I}{L[1-\bar{K}]}$.

In the above equation, $\bar{K}, K, E, I$ and $L$ are normalised rigidity, spring torsional stiffness, beam's Young's modulus, beam's moment of inertia, and beam length, respectively. The normalised rigidity $\bar{K}$ is dependent on the type of joint and the beam aspect ratio. The values of $\bar{K}$ with respect to beam aspect ratio have been plotted in the literature [16]. In the current work, the value of $\bar{K}$ has been used based on entangled and crossed joints as presented in [16].

In the current work, the random network of nanoropes (also known as bundles) has been generated by scripting an algorithm (refer to the supplementary material where the developed code is provided), wherein a combination of stretched and wavy (un-stretched) CNT nanoropes is assumed to be the content of a typical buckypaper [34, 35]. In the buckypaper, the stretched and un-stretched nanoropes have been represented as straight lines and sine curves, respectively $[15,180]$. The steps involved in the automated distribution of nanoropes are given below:

- Step 1: The total number, amplitude, and period of sine curves have been defined. The total number and length of straight lines are also defined. The length of straight lines is defined as the same as the period of sine curves. An initial sine curve has been created in the first instance, and then, it has been randomly copied into multiple locations and randomly rotated in each location. Several points have been defined on the sine curves and the straight lines to generate finite-element-based nodes.

- Step 2: The points that intersect between curves and straight lines have been identified using the intersection function available in the Matlab library. At the location of these points, the curves and straight lines have been split to create new finite-element nodes. To locate these points in the data generated in Step 1, a user-defined function findposition has been created. A second userdefined function insertrows has been developed to insert extra points(nodes) into the data generated in Step 1, as a result of intersections.

- Step 3: A text file containing the data created in Step 1 and Step 2 has been written as an output of the Matlab code. This text file contains the node, beam element, and spring element data. Each sine curve and the straight line have been represented by 20 nodes and 19 beam elements. The number of nodes and elements has been left open to modification to take into account intersection points. At the intersection points, torsional spring elements have been created.

- Step 4: The above three steps have been repeated for different stretched and un-stretched ratios of nanoropes to generate five different buckypapers (refer to Fig. 3a-e).

The Matlab code explained above generates an output text file containing the necessary finite-element input data of buckypapers (involving the definition of nodes, beam elements, and torsional spring elements). The data are saved with ".inp" extension. At the next stage, within the finiteelement code ABAQUS [40], the beam elements and spring elements are modelled using the element types B21 and Spring2, respectively.

To model the buckypaper-Bismaleimide (BMI) composite system, it is assumed that the BMI matrix is a continuous structure. Therefore, the matrix is modelled by general purpose 3D triangular shell elements of ABAQUS (S3) [40]. The buckypaper is considered to be sandwiched between two layers of the BMI matrix. Here, two layers of shell elements are modelled on either side of the beam network to represent a $60 \%$ fibre weight fraction. The interface between the buckypaper and the BMI matrix is assumed to be perfectly bonded and modelled as a rigid contact using Tie constraint. The BMI matrix is considered to be isotropic with Young's modulus and Poisson's ratio as $4.6 \mathrm{GPa}$ and 0.33 , respectively [101]. The sectional view of a mesh of buckypaper composite is shown in Fig. 3f, where the yellow background represents the solid shell mesh of resin. The calculated mechanical properties of buckypaper composites using the model developed here are presented in Sect. 3.5.

\subsubsection{Supplementary note: Waviness of nanoropes in the buckypaper}

The wavy curvatures of nanoropes in the buckypapers are evident in several SEM images presented in the literature $[34,35,96,121]$. The curvatures in the geometry of waves can be represented by various functions, including sine, cosine, and exponential. The arbitrary waviness of ropes in the buckypaper might not exactly follow any of these functions. However, various works have successfully 
approximated and validated sine $[15,180]$ and cosine functions [17, 48, 48]. The current work is based on sine approximations presented by Berhan et al. [15]. Due to the convenience of representing nanorope waviness by period, amplitude, and frequency of sine waves, these functions have been chosen here. Furthermore, the degree of waviness can be varied by modifying the frequency and period of sine functions. Other means of modelling curvature include waviness factor [47] and a waviness parameter [68, 69]. Feng et al.'s [47] model used a waviness factor $\left(\gamma=l_{\mathrm{nt}} / l_{\mathrm{f}}\right)$ to determine the influence of nanorope curvature on CNT composite film modulus, wherein $l_{\mathrm{nt}}$ and $l_{\mathrm{f}}$ were actual length and distance between two ends of nanoropes, respectively. The straight and wavy nanoropes lead to $\gamma=1$ and $\gamma>1$, respectively. Tsai et al. [68] assumed a sine curve to represent wavy nanorope and defined the waviness parameter as $w=A / L$, wherein $A$ and $L$ were the amplitude and wavelength, respectively. The overarching aim of the research community in this field is to minimize waviness and maximize the straightness of nanoropes, since the presence of nanorope waviness leads to a lower buckypaper mechanical property. The waviness can be significantly reduced by methods such as stretching $[34,35]$.

\subsection{Level 6: Simulation of an automotive suspension control arm}

The broader objective of the current work is to introduce graphene-based materials into critical industry-grade structural components for enhanced mechanical performance. As discussed in the preceding sections, neither graphene nor CNT in their nanometer size scales can be directly cast or fabricated into a geometrically complex macro-scale engineering component. Note that the powder format of graphene or CNT nanomaterials does not offer essential mechanical properties. However, the paper variants such as graphene paper or buckypaper can play the role of primary reinforcement material in high-performance engineering components at macro-scale. Therefore, an effort has been made here to demonstrate the suitability of buckypaper as a
Fig. 4 Evolution of lightweight buckypaper-reinforced composite concept. a A control arm with four hardpoints. $\mathbf{b}$ Partitioning of the control arm. c Alternating slices of SMC, carbon prepreg, and buckypaper

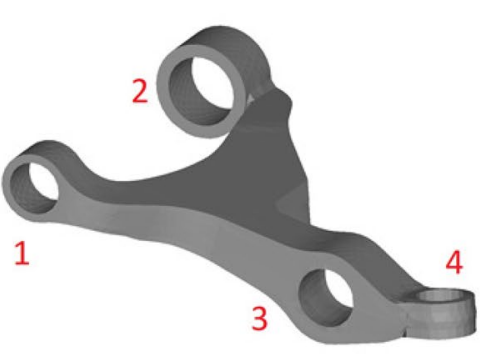

(a)

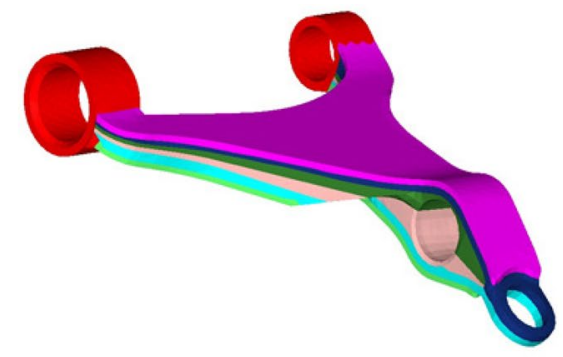

(b)

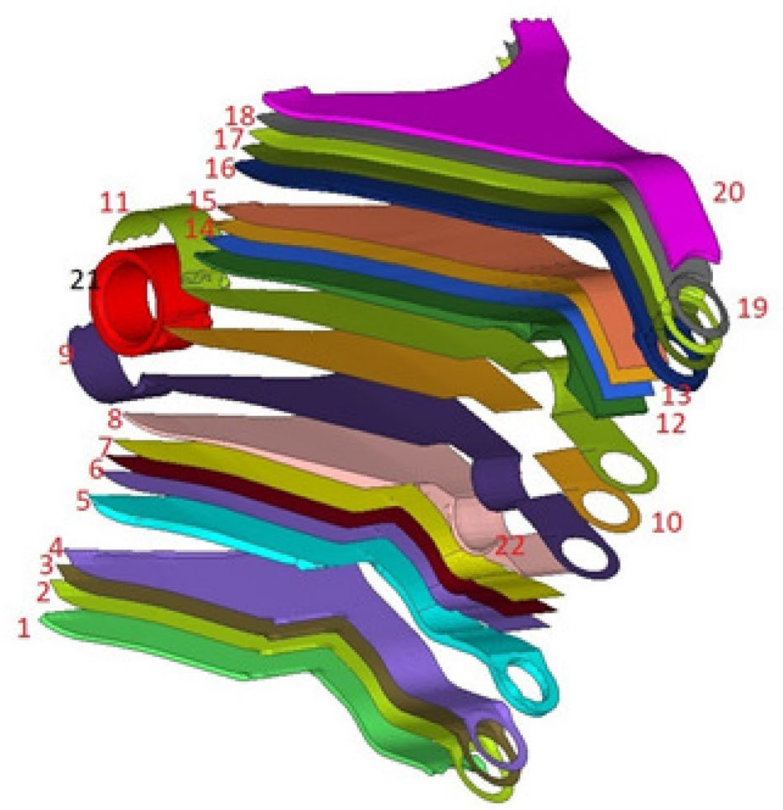

(c) 
reinforcing layer in composite automotive suspension control arms. The evolution of the buckypaper reinforced composite automotive suspension control arm concept is shown in Fig. 4. A typical control arm (refer to Fig. 4a) of a road vehicle suspension weighing $4.2 \mathrm{~kg}$ has been chosen for a baseline design. This baseline design is assumed to be cast using an aluminium alloy. The current work aims to improve this baseline design by replacing aluminium alloy with a buckypaper-based material system.

A multi-material system consisting of the sheet moulding compound (SMC), carbon fibre prepreg, and buckypaper composite has been assembled to form the geometry of control arm (refer to Fig. 4b). The buckypaper composite of MWCNT (multi-wall carbon nanotube) and bismaleimide (BMI) resin, as described in the preceding paragraph, is used in the control arm. This composite buckypaper offers a density of $1.36 \mathrm{gm} / \mathrm{cm}^{3}$ (based on $60 \%$ fibre weight fraction) and Young's modulus of $370.0 \mathrm{GPa}$ (refer to Sect. 3.5). Thickness of the composite sheet is $50 \mu \mathrm{m}$, which is calculated based on $60 \%$ fibre weight fraction configuration. The SMC chosen is AMC8592, which offers a density of $1.48 \mathrm{gm} / \mathrm{cm}^{3}$ and Young's modulus $69.0 \mathrm{GPa}$. The carbon fibre composite chosen is a prepreg with grade HM508552 (unidirectional fibre composite). The resin in this prepreg is Hexply-8552-epoxy, and the fibre volume fraction is $60 \%$. The thickness of this prepreg is $0.13 \mathrm{~mm}$, and the density is $1.5 \mathrm{gm} / \mathrm{cm}^{3}$.

An isometric view of the multi-material concept control arm is shown in Fig. 4b. In this isometric view, six slices visible in pink, blue, dark green, cream, light blue, and green colours are the sections of the SMC. The two rings shown in red colour are also made of the SMC. A split view showing all different materials used in the multi-material control arm is presented in Fig. 4c. This view shows 21 separate components and the material selection for each component is shown in Table 2. This results in ten sheets of prepreg and five sheets of buckypaper. The view in Fig. $4 \mathrm{~b}$ is of the consolidated part, wherein prepreg and buckypaper become hidden underneath, and only the SMC becomes visible in the image. Since the stiffness and strength properties of prepreg and buckypaper are directional, each prepreg sheet is designed as a laminate composed of eight plies oriented at $0^{\circ}, 45^{\circ}, 60^{\circ}$, and $90^{\circ}$, repetitively. Also, each buckypaper sheet is designed as a laminate of 16 plies oriented at

Table 2 Components in the concept design of control arm and the corresponding materials (refer to Fig. 4)

\begin{tabular}{ll}
\hline Material used & Part number \\
\hline Sheet moulding compound (SMC) & 151216202122 \\
Prepreg & 246891113151719 \\
Buckypaper & 37101418 \\
\hline
\end{tabular}

$0^{\circ}, 45^{\circ}, 60^{\circ}$, and $90^{\circ}$, repetitively. Such an arrangement of layers of buckypapers and prepregs is made to attain near isotropic two-dimensional material properties. Furthermore, adequate shear properties are also attained due to such laminated composite layers. The two-dimensional arrangements of buckypapers and prepregs in conjunction with bulking of sheet moulding (SMC) compound would offer the resulting component adequate mechanical properties in all directions. Within the finite-element model, the laminates are represented by the conventional shell elements and the SMC is represented by solid elements.

In the current investigation, mechanical performance of the automotive suspension arm is assessed through vibrational analysis and static analysis for demonstration. Since a control arm undergoes motion only in a few directions, its degrees of freedom are restricted to those few directions. The vibrational analysis involves extracting mode shapes that are relevant to these degrees of freedoms. The mode shapes that need to be extracted are typically vertical bending modes, in-plane bending modes, out-of-plane bending modes, and torsional modes. The mode shapes and their natural frequencies are highly influenced by the component's material properties, geometry of the component design, and the component boundary conditions. Changing the material of a component does not change its mode shape, while the natural frequency will be changed. In automotive industry, the common practice is to extract the first five mode shapes of suspension components along with the corresponding natural frequencies and benchmark them. The benchmarking is usually performed against predetermined targets or baseline designs. The baseline design can be equivalent to an existing similar vehicle manufactured in house or by a competitor firm. The aim of such modal analysis in component design is normally to ensure an increase in the natural frequency of the component compared to benchmark natural frequencies. The higher natural frequency indicates higher stiffness, as well. In the finite-element analysis, the mode shapes are the eigenvectors, and the natural frequencies are the eigenvalues, obtained by solving the equation of motion, as described below

$\left([\mathbf{K}]-\omega^{2}[\mathbf{M}]\right)\{\mathbf{x}\}=\{\mathbf{0}\}$.

In the above equation of motion, $[\mathbf{K}],[\mathbf{M}], \omega$, and $\mathbf{x}$ are stiffness matrix, mass matrix, frequency of vibration, and displacement vector, respectively. There are various finiteelement-based algorithms available to extract eigenvectors and eigenvalues from the above equation of motion. The algorithms include Lanczos, block Lanczos, automated multi-level sub-structuring, Givens, Householder, inverse power, Sturm modified inverse power, and subspace methods. In the current work, the block Lanczos method has been used to extract mode shapes and natural frequencies of the 
conceptual and baseline design of the control arm. Besides dynamic analysis, checking the limits of deflections under standard loading conditions is also crucial. Thus we have investigated the performance of composite control arms under the static loading condition. For carrying out the static analysis, the effect of inertia is neglected and using the same stiffness matrix [K], the standard system of equations represented by $\{\mathbf{F}\}=[\mathbf{K}]\{\mathbf{x}\}$ is solved, where $\{\mathbf{F}\}$ represents the nodal force vector. The numerical analyses of the baseline and the concept composite design of control arm are presented in Sect. 3.6.

\subsubsection{Supplementary note: Conceptual design of the automotive control arm}

The baseline design shown in Fig. 4a is a CAD model based on a C-segment automobile's existing aluminium control arm. Detailed geometrical features have been removed for the sake of simplicity. The current work aims to replace this baseline metallic design with a multi-material design, wherein the buckypaper would act as a reinforcement. The concept shown in Fig. $4 \mathrm{c}$ is a result of prior parametric studies. These parametric design studies considered several different concepts. An initial trial involved slicing off the baseline control arm (refer to Fig. 4a) into two sections and then placing a buckypaper/prepreg layer at the centre. However, this design involving two SMC sections and one buckypaper/ prepreg laminate did not offer the required mechanical performance. Therefore, several iterations have been carried out by progressively increasing slices of SMC and buckypaper/ prepreg laminates. The iterations also varied the number of plies within each laminate based on manual optimisation of ply-stacking sequences. We settled with the arrangement of slices shown in Fig. 4c, since it offered a mechanical performance higher than that of the baseline design. The manual optimisation of ply-stacking sequences concluded the prepreg laminates composed of eight plies repeated at $0^{\circ}, 45^{\circ}, 60^{\circ}$ and $90^{\circ}$, and buckypaper laminate of 16 plies repeated at $0^{\circ}, 45^{\circ}, 60^{\circ}$, and $90^{\circ}$ as the optimum stacking sequence.

\subsubsection{Supplementary note: Role of SMC and prepreg}

Although the main aim of the current work is to demonstrate the suitability of buckypaper as a raw material to manufacture an engineering component, for the automotive component chosen here, the buckypaper on its own cannot fulfil all the design needs. For instance, the buckypaper may not conform to the intricate geometric features of the control arm. As a result, the sheet moulding compound (SMC) with the ability to be moulded to complex geometric shapes has been selected as a part of the multi-material component design. Furthermore, the resin of the buckypaper composite may not interact effectively with the SMC. Therefore, a carbon fibre-epoxy prepreg has been selected, since the epoxy resin has the ability to strongly bond to various types of materials. Such firm adhesive bonding properties have been historically validated [97] and accepted industry-wide. SMC is a layered composite system involving a polymeric matrix reinforced by chopped long fibres. The SMC sheets on rollers are available to be purchased from various composite suppliers, including Hexcel Inc and Toray Inc. The gel-like texture of the SMC facilitates this raw material to
Fig. 5 An illustration of the proposed multi-material system. Here, the resin is not shown in the images of prepreg and buckypaper. Both prepreg and buckypaper are used in composite format for the design of control arm

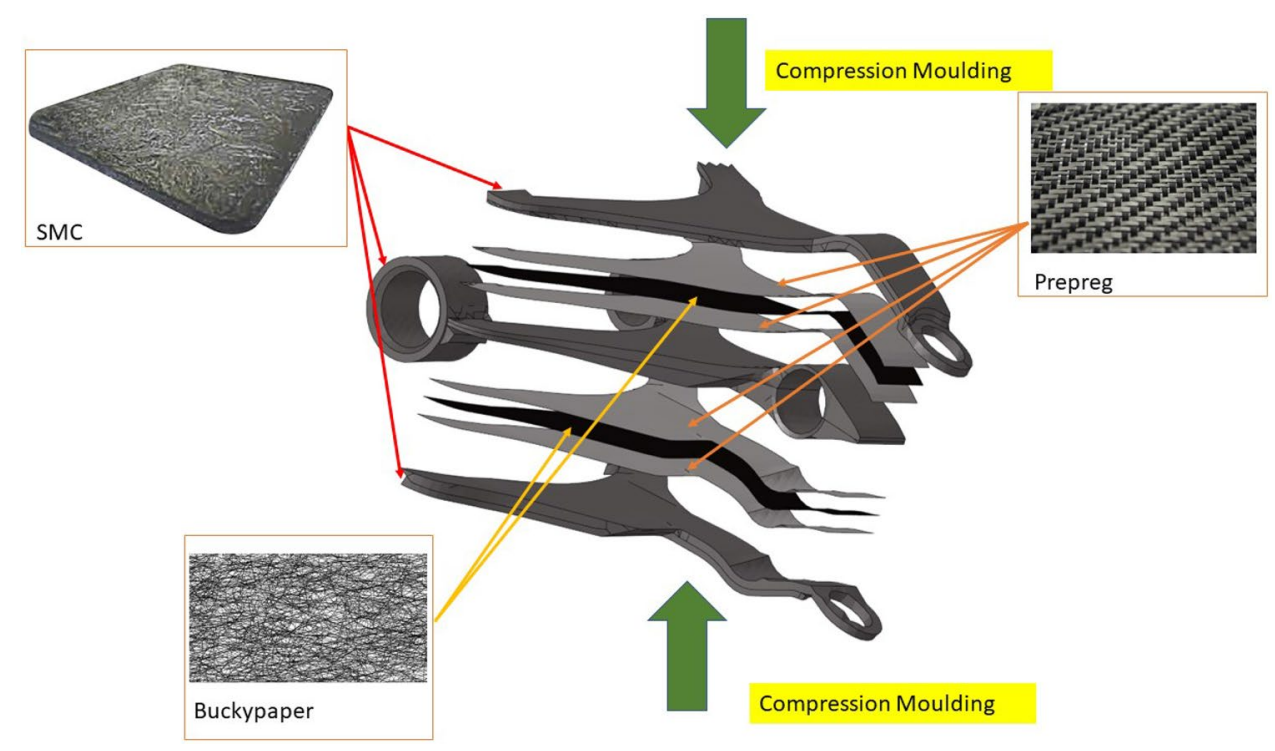


be compression moulded into complex geometries. The grade of SMC chosen in the current work is AMC8592. This grade is composed of chopped carbon fibres with $1^{\prime \prime}$ length at $53 \mathrm{wt} \%$ and vinyl-ester resin [129]. A prepreg is a composite system of continuous fibres and polymeric resin. Uncured continuous sheets of prepreg are supplied on rollers by raw material suppliers, including Hexcel Inc and Toray Inc. These materials can be laminated, cured, and moulded into engineering components. The grade of prepreg used in the current work is HM508552. This prepreg involves unidirectional carbon fibres at $60 \mathrm{vol} \%$ and epoxy resin [67]. Compression moulding of carbon fibre prepreg and carbon fibre SMC into complex shapes has been demonstrated by the research community widely [175]. The moulding of the proposed multi-material control arm is illustrated in the Fig. 5. Here, the slices of SMC and the laminated layers of prepreg and buckypaper can be assembled and compression moulded using the process demonstrated by Wulfsberg et al. [175].

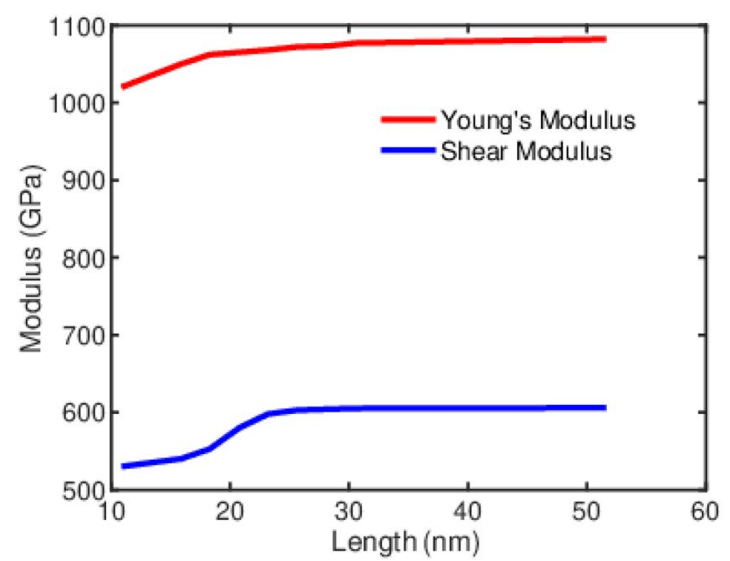

(a)

\section{Results and discussion}

In this section, we have systematically presented the numerical results for investigating the mechanical behaviour of all the carbon-based structural components at multiple length scales, starting from graphene to the buckypaper composites.

\subsection{Mechanical properties of graphene}

An atomistic finite-element representation of the graphene sheet can be seen in Fig. 1a. First, the atomistic finite element models of various sizes have been constructed to identify the sensitivity of Young's modulus to the sheet dimensions. To calculate the effective Young's modulus, the graphene sheet has been constrained at one edge, and a load is applied on the opposite edge. The resulting tensile sheet deflection has been used to calculate Young's modulus using the relation

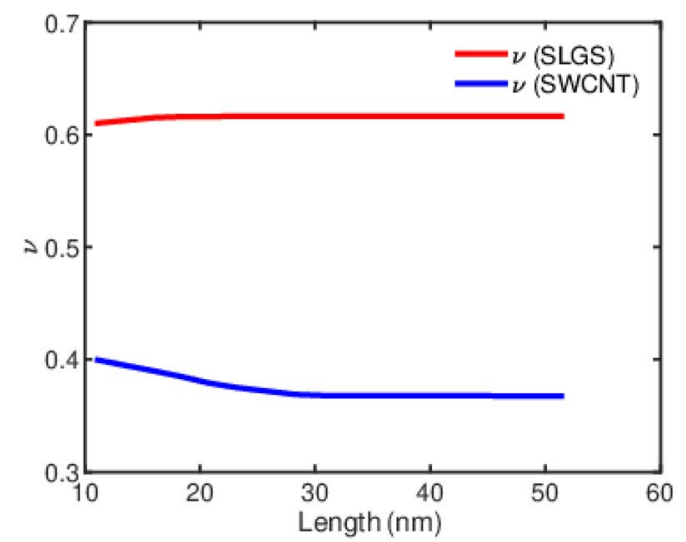

(b)

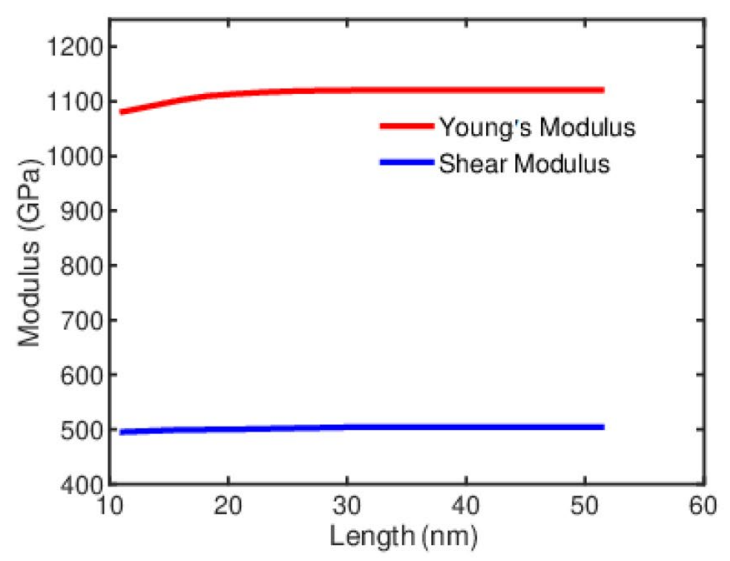

(c)

Fig. 6 Computation of mechanical properties of graphene and carbon nanotube. a Variation of Young's modulus and shear modulus of graphene with respect to the sheet length. b Variation of Poisson's ratio of SWCNT and SLGS with respect to the length. $\mathbf{c}$ Variation of Young's modulus and shear modulus of SWCNT with respect to the tube length 
Table 3 Graphene (SLGS): computed Young's modulus $E$ (GPa) along with comparative results from existing literature

\begin{tabular}{ll}
\hline Analysis type & $E(\mathrm{GPa})$ \\
\hline Numerical & $1030.0[88] 990.0[107] 1000.0$ [54] 1450.0 [146] 1130.0 [176] 350.0 [14] 740.0 [56] \\
& $1190.0[100]$ \\
& $1600.0[166] 1200.0[5] 2900.0$ [151] 860.0 [57] 810.0 [104] 1400.0 [131] 420.0 [77] \\
Experimental & $200.0[157] 900.0[98]$ \\
Present (numerical) & 1082.0 \\
\hline
\end{tabular}

Table 4 Graphene (SLGS): computed shear modulus $G$ (TPa) along with comparative results from existing literature

\begin{tabular}{lc}
\hline Analysis type & $\mathrm{G}(\mathrm{TPa})$ \\
\hline Numerical & $490.0[88] 400.0[70]$ \\
& $300.0[137] 230.0$ \\
{$[142] 210.0[147]$} \\
$500.0[180]$ \\
$500.0[14] 400.0[56]$ \\
$180.0[91] 210.0$ \\
{$[162] 450.0[59]$} \\
$720.0[53]$ \\
Present (numerical) & 606.0 \\
\hline
\end{tabular}

$E=\frac{F L}{A \delta}$.

In the above equation, $E, F, L, A, \delta$ are Young's modulus of graphene, applied force, sheet length, sheet cross-section area, and computed sheet deflection, respectively. The variation of the $E$ with respect to the sheet length is shown in Fig. 6a. At smaller lengths, the modulus increases with the length due to the prominence of boundary effect. However, at larger lengths, the modulus becomes constant with respect to the length of the periodic nanostructure. The Young's modulus at larger lengths is $1082 \mathrm{GPa}$ and is comparable with the literature, as shown in Table 3.

To measure shear modulus using atomistic simulations, one edge of the graphene sheet has been constrained, and the opposite edge has been subjected to shear force. The resulting shearing deflection has been recorded to calculate shear modulus as

$G=\frac{F}{A \times \tan ^{-1}\left[\frac{\delta s}{l}\right]}$.

In the above equation, $G, F, l, A, \delta s$ are the shear modulus, applied shear force, sheet length parallel to the shearing direction, sheet cross-section area, and computed sheet deflection, respectively. The variation of the $G$ with respect to the sheet length is shown in Fig. 6a. At smaller lengths, the shear modulus increases with the length due to boundary
Table 5 Graphene (SLGS): computed Poisson's ratio $v$ along with comparative results from existing literature

\begin{tabular}{ll}
\hline Graphene type & $v$ \\
\hline Numerical & $1.4[142] 0.29[146] 0.72[54]$ \\
& $0.22[55] 0.23[185] 0.11[4]$ \\
& $0.05[142] 0.06[86] 0.08$ \\
Present (numerical) & {$[181] 0.52[57]-0.001[53]$} \\
\hline
\end{tabular}

Table 6 SWCNT: Computed Young's modulus $E(\mathrm{GPa})$ along with comparative results from existing literature

\begin{tabular}{lc}
\hline Analysis type & $E(\mathrm{GPa})$ \\
\hline Experimental & $980.0[99] 1300.0[79] 1470.0[179]$ \\
Numerical & $1000.0[88] 1200.0[95] 5300.0[9]$ \\
& $2570.0[125] 2900.0[147] 760.0$ \\
& {$[112] 2030.0[110]$} \\
& $860.0[103] 1320.0[133] 940.0$ \\
& {$[135] 2150.0[188] 2150.0[188]$} \\
Present (numerical) & $100.0[51] 1050.0[148]$ \\
& 1080.0
\end{tabular}

effect. However, at larger lengths, the shear modulus of the periodic structure remains constant with respect to the length. The shear modulus at larger lengths is $606 \mathrm{GPa}$ and it is in agreement with the literature (refer to Table 4).

Using the atomistic simulations, Poisson's ratio of graphene sheet has been calculated by introducing tensile strain at one edge and then recording the longitudinal and lateral strains. Poisson's ratio is the negative ratio between lateral and longitudinal strains. The computed Poisson's ratio for graphene sheets is presented in Fig. 6b. The Poisson's ratio computed for a single-layer graphene sheet here is 0.62 . This value has been compared against the available literature in Table 5. wherein a rather large range of values can be noticed. However, the presently computed Poisson's ratio falls well within the range.

\subsection{Mechanical properties of SWCNT}

The mechanical properties predicted for SWCNTs are found to be exceptionally high. However, these mechanical 
Table 7 SWCNT: computed $G$ (GPa) compared against existing literature

\begin{tabular}{ll}
\hline Analysis type & $\mathrm{G}$ \\
\hline Numerical & $480.0[76] 500.0[45] 140.0[125] 500.0[33] 460.0[46] 410.0[152]$ \\
& $420.0[153] 500.0[10] 340.0[173] 470.0[100] 320.0[103] 510.0$ \\
Present (numerical) & {$[134] 500.0[58] 900.0[148]$} \\
\hline
\end{tabular}

Table 8 SWCNT: predicted Poisson's ratio $v$ compared against existing literature

\begin{tabular}{ll}
\hline Analysis type & $v$ \\
\hline Numerical & $0.35[9] 0.65[183] 0.45[7] 0.96[8]$ \\
& $0.65[185] 0.28[43] 0.06[148] 0.32$ \\
& {$[158]$} \\
Present (numerical) & 0.37 \\
\hline
\end{tabular}

properties cannot be retained at higher scales, such as that in buckypaper. The Young's modulus, shear modulus, and Poisson's ratio computed in the current work have been compared against the literature in Tables 6, 7 and 8, wherein these are found to be well within acceptable limits.

In the atomistic simulations, the Young's modulus of SWCNT has been calculated by constraining the tube's one end, applying tensile force at the tube's opposite end and then using the resulting deflection in Eq. 10. The variation of $E$ with respect to the tube length is shown in Fig. 6c. The value of $E$ computed for the tube length of $54 \mathrm{~nm}$ is $1080 \mathrm{GPa}$. It is important to note that a similar Young's modulus value has been offered by SLGS, as shown in Sect. 3.1. The value of $E$ obtained in the current work has been compared against the values presented in the literature (refer to Table 6), where value of $E$ up to $5300 \mathrm{GPa}$ has been reported. Avila and Lacerda [9] presented such a large value based on the tensile analysis of an SWCNT zigzag $(14,0)$ configuration with tube length $2.4 \mathrm{~nm}$. In the literature (Table 6), the lowest value of $E$ is $100 \mathrm{GPa}$, presented by Ge et al. [51]. They modelled a SWCNT of $1 \mathrm{~nm}$ diameter and measured Young's modulus in the radial direction, resulting in the lowest magnitude. As per the literature, $E$ varies from 100 to $5300 \mathrm{GPa}$. The Young's modulus computed in the current work is $1080 \mathrm{GPa}$ (Table 6) and falls well inside this range.

The shear modulus of the SWCNT has been calculated using the principle of torsional rigidity. A similar method has also been implemented by Zaeri et al. [180]. One end of the SWCNT has been constrained, a torsional load has been applied to the other end, and the resulting tangential deflection has been recorded. The shear modulus of SWCNT can be calculated by

$G=\frac{T l}{J \delta \theta}$
In the above equation, $T$ is the torque, $l$ is the length of SWCNT, $\delta \theta$ is the twist angle, and $\mathrm{J}$ is the polar moment of inertia. The variation of shear modulus $G$ with respect to the SWCNT length is shown in Fig. 6c. As per this plot, $G$ does not tend to be influenced by the tube length. The $G$ here is found to be $504 \mathrm{GPa}$, which can be noted to be significantly different compared to that of SLGS. The computed value of $G$ in the current work has been compared against the literature in Table 7. As per the literature, the shear modulus of SWCNT can be as high as $900 \mathrm{GPa}$. This indicates that SWCNT can offer shear stiffness more than 11 times higher than that of structural steel. Schiebold and Mehner [148] predicted the shear modulus to be $900 \mathrm{GPa}$ by considering an equivalent cylindrical solid mesh of SWCNT with $0.5 \mathrm{~nm}$ diameter. As per the literature, the shear modulus $(G)$ of SWCNT can be as low as $320.0 \mathrm{GPa}$ [103], where a springbased finite-element model of SWCNT was used with $2 \mathrm{~nm}$ diameter. It can be concluded that the $G$ of SWCNT ranges from 320 to $900 \mathrm{GPa}$ in the literature. The computed value $504 \mathrm{GPa}$ in the current work falls well within this range.

Poisson's ratio $(v)$ has been measured by introducing tensile forces to the SWCNT, followed by recording transverse and axial strains. The computed Poisson's ratio as a function of SWCNT length is shown in Fig. 6b. At lower lengths, Poisson's ratio drops with the increase in length up to $25 \mathrm{~nm}$. At higher lengths (i.e., $>25 \mathrm{~nm}$ ), $v$ is found to be independent of the SWCNT length. It can be concluded that the $v$ remains stable at 0.37 at lengths beyond $25 \mathrm{~nm}$. Therefore, this value can be used to model high aspect ratio SWCNTs within a buckypaper. The computed value of Poisson's ratio $(v)$ has been compared against literature in Table 8. As per the literature, $v$ of SWCNT can be as low as 0.06. Such a low value has been reported by Domínguez-Rodríguez et al. [43] for a SWCNT with diameter $\approx 0.2 \mathrm{~nm}$. The largest value of $v$ identified in the literature (Table 8) is 0.96 . Askari and Ghasemi-Nejhad [7] determined the $v$ to be 0.96 with the aid of a space frame representation of SWCNT. The element type used to represent $\mathrm{C}-\mathrm{C}$ bonds in this analysis was linktype instead of equivalent beams used in the current work. As per the literature data presented in Table $8, v$ can range between 0.06 and 0.96 . The computed value of 0.37 in the current work falls well within this range. 
Table 9 MWCNT: computed Young's modulus $E$ (GPa) along with comparative results from existing literature

\begin{tabular}{llll}
\hline Analysis type & $E(\mathrm{GPa})$ & Total walls & Source \\
\hline Numerical & 1100.0 & 2 & {$[87]$} \\
Numerical & 1120.0 & 3 & {$[87]$} \\
Numerical & 1120.0 & 4 & {$[87]$} \\
Numerical & 1390.0 & 2 & {$[73]$} \\
Numerical & 1500.0 & 3 & {$[73]$} \\
Numerical & 1600.0 & 4 & {$[73]$} \\
Numerical & 1170.0 & 2 & {$[183]$} \\
Numerical & 1190.0 & 3 & {$[183]$} \\
Numerical & 1200.0 & 4 & {$[183]$} \\
Numerical & 1050.0 & 2 & {$[18]$} \\
Numerical & 953.1 & 2 & {$[111]$} \\
Numerical & 1045.0 & 2 & {$[134]$} \\
Numerical & 1148.0 & 3 & {$[134]$} \\
Numerical & 1050.0 & 4 & {$[134]$} \\
Numerical & 1050.0 & 5 & {$[134]$} \\
Numerical & 1206.0 & 2 & Present \\
Numerical & 1292.0 & 3 & Present \\
Numerical & 1354.0 & 4 & Present \\
Numerical & 1401.0 & 5 & Present \\
\hline
\end{tabular}

\subsection{Mechanical properties of MWCNT}

The finite-element models of CNTs with up to five walls have been considered in the current work. The variation and sensitivity of $E$ to the number of walls in a MWCNT are presented in Fig. 7a. In the current analysis, $E$ of SWCNT is found to be $1080.0 \mathrm{GPa}$, whereas it is $1206.0 \mathrm{GPa}$ for a MWCNT with double walls. For the MWCNTs with three, four, and five walls, $E$ is found to be 1292.0, 1354.0, and $1401.0 \mathrm{GPa}$, respectively. There is a significant increase in the modulus of double wall carbon nanotubes (DWCNT) compared to SWCNT. Beyond two walls, the slope of this curve is not significantly high. The computed $E$ of MWCNTs for various numbers of walls has been compared against the literature data in Table 9. From the literature, it is evident that $E$ of MWCNTs can be up to $1600 \mathrm{GPa}$. This means MWCNTs can be 7.5 times stiffer compared to structural steel under tension. Table 9 indicates that the $E$ of MWCNT can range from 953.1 to $1600.0 \mathrm{GPa}$. The computed $E$ of MWCNTs in the current work falls well within this range.

The shear modulus $G$ of MWCNTs has been calculated by applying torque and then using Eq. 12. The variation of $G$ with respect to the number of MWCNT walls is shown in Fig. 7a. This plot follows a similar trend as that of $E$ for the MWCNTs. The $G$ of DWCNT is found to be significantly higher compared to that of SWCNT. It can also be observed that the $G$ increases with the number of walls. However, the rise in $G$ from four-walled CNT to five-walled CNT is
Table 10 MWCNT: computed shear modulus $G$ (GPa) along with comparative results from existing literature

\begin{tabular}{llll}
\hline Analysis type & $G(\mathrm{GPa})$ & Total walls & Source \\
\hline Numerical & 400.0 & 2 & {$[87]$} \\
Numerical & 360.0 & 3 & {$[87]$} \\
Numerical & 360.0 & 4 & {$[87]$} \\
Numerical & 400.0 & 2 & {$[73]$} \\
Numerical & 450.0 & 3 & {$[73]$} \\
Numerical & 450.0 & 4 & {$[73]$} \\
Numerical & 418.0 & 2 & {$[18]$} \\
Numerical & 514.0 & 2 & {$[134]$} \\
Numerical & 556.0 & 2 & Present \\
Numerical & 598.0 & 3 & Present \\
Numerical & 622.0 & 4 & Present \\
Numerical & 638.0 & 5 & Present \\
\hline
\end{tabular}

found to be moderate. The $G$ of MWCNTs with two, three, four, and five walls are found to be 556.0, 598.0, 622.0, and $638.0 \mathrm{GPa}$, respectively. These computed values have been compared against the literature data in Table 10. Note that the literature data are based on only two-, three-, and fourwalled CNTs. The literature suggests that the $G$ of MWCNTs can range from 360.0 to $514.0 \mathrm{GPa}$, which is in good agreement with the current predictions.

Poisson's ratio $(v)$ has been measured by introducing tensile forces to the MWCNT, followed by recording the transverse and axial strains. The computed Poisson's ratio as a function of the number of MWCNT walls is shown in Fig. 7b. According to this plot, as the number of walls is increased, $v$ drops initially and then converges. The drops in $v$ from SWCNT to DWCNT and from DWCNT to triplewalled CNT are considerable. However, the drops in $v$ from triple-walled CNT to four-walled CNT and from four-walled CNT to five-walled CNT are negligible. Therefore, it can be assumed that Poisson's ratio $v$ remains constant when the number of walls is increased beyond three. The Poisson's ratio $v$ for double-walled, triple-walled, four-walled, and five-walled MWCNTs are found to be $0.35,0.33,0.326$, and 0.324 , respectively. These data have been listed along with the values from literature in Table 11. Zhang et al. [183] presented a value of 0.42 for an armchair DWCNT with $3 \mathrm{~nm}$ diameter. From Table 11, it is evident that the values of $v$ for MWCNTs computed in the current work are comparable to the one presented in published literature.

\subsection{Mechanical properties of MWCNT bundle}

In the current work, four different configurations of the MWCNT bundles have been investigated. These include a bundle with two MWCNTs (configuration(2), refer to Fig. 1d), a bundle with three MWCNTs (configuration(3), refer to 


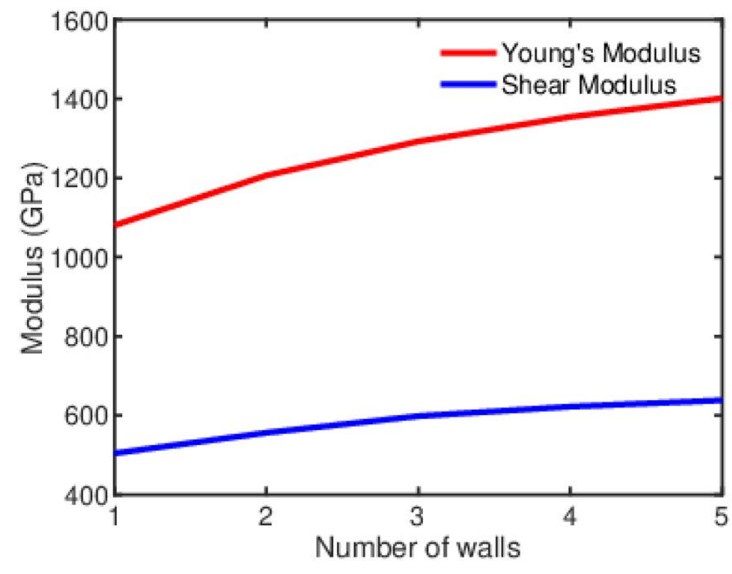

(a)

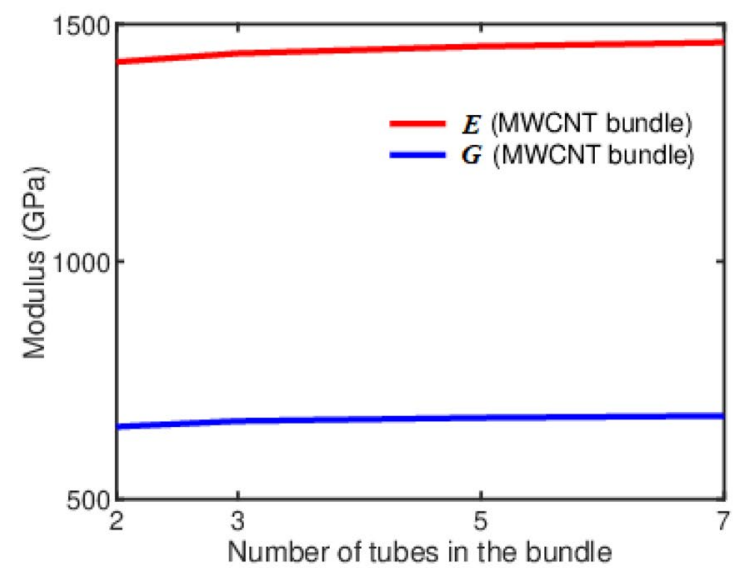

(c)

Fig. 7 Computation of mechanical properties of MWCNT and MWCNT bundles. a Variation of Young's modulus and shear modulus of MWCNT with respect to the number of walls. b Variation of Poisson's ratio of MWCNT with respect to the number of walls. $\mathbf{c}$

Table 11 MWCNT: computed Poisson's ratio $v$ along with comparative results from existing literature

\begin{tabular}{llll}
\hline Analysis type & $v$ & Total walls & Source \\
\hline Numerical & 0.42 & 2 & {$[183]$} \\
Numerical & 0.35 & 2 & Present \\
Numerical & 0.33 & 3 & Present \\
Numerical & 0.326 & 4 & Present \\
Numerical & 0.324 & 5 & Present \\
\hline
\end{tabular}

Fig. 1e), a bundle with five MWCNTs (configuration(5), refer to Fig. 1f), and a bundle with seven MWCNTs (configuration(7), refer to Fig. 1g). The Young's modulus $(E)$ has been recorded by introducing boundary conditions same as in Sect. 3.2 and using Eq. 10. The $E$ for configuration(2), configuration(3), configuration(5), and configuration(7) of MWCNTs

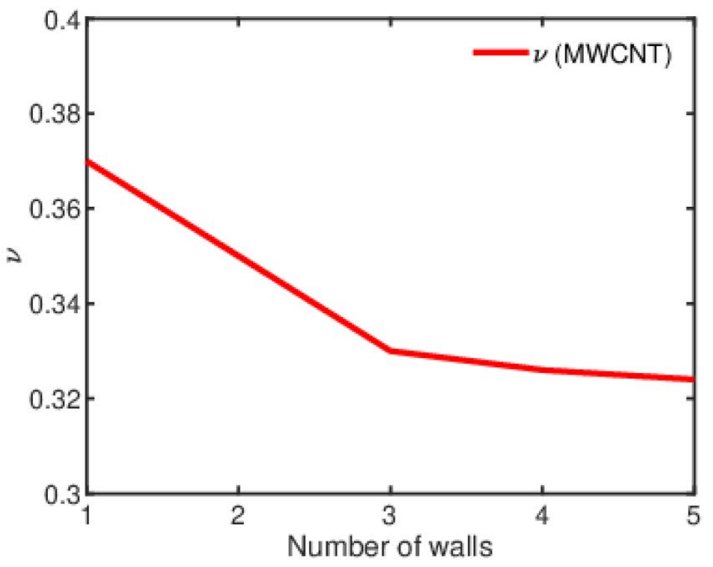

(b)

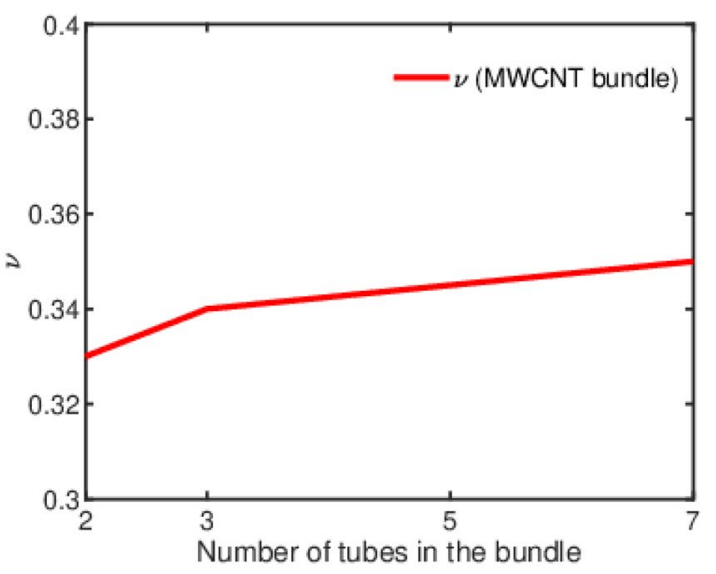

(d)

Variation of Young's modulus and shear modulus of MWCNT bundles with respect to the number of MWCNT tubes. d Variation of Poisson's ratio of glsMWCNT bundles with respect to the number of MWCNT tubes

Table 12 MWCNT bundles: computed Young's modulus E (GPa) along with comparative results from existing literature

\begin{tabular}{llll}
\hline Analysis type & $E(\mathrm{GPa})$ & Total walls & Source \\
\hline Numerical & 850.0 & 7 & {$[92]$} \\
Numerical & 1058.0 & 2 & {$[11]$} \\
Numerical & 840.0 & 3 & {$[11]$} \\
Numerical & 747.0 & 7 & {$[11]$} \\
Experimental & 15.0 & Few & {$[170]$} \\
Experimental & 74.0 & $>100$ & {$[182]$} \\
Experimental & 24.0 & $>100$ & {$[108]$} \\
Analytical & 633.0 & N/A & {$[94]$} \\
Analytical (SWCNT) & 1400.0 & N/A & {$[164]$} \\
AFM & 1310.0 & 7 & {$[143]$} \\
Numerical & 1420.0 & 2 & Present \\
Numerical & 1453.0 & 3 & Present \\
Numerical & 1461.0 & 7 & Present \\
\hline
\end{tabular}


are found to be $1420,1438,1453$, and $1461 \mathrm{GPa}$, respectively. This indicates a marginal increase in stiffness with the addition of tubes. The variation of $E$ with respect to the number of MWCNTs in a bundle is shown in Fig. 7c. The slope of the curve is not significant in this plot. The computed value of $E$ for MWCNT bundles has been compared against literature in Table 12. Liu et al. [92] used a combination of atomistic and continuum mechanics to predict $E$ of SWCNT bundles, while Bai et al. [11] fabricated several $\mathrm{cm}$ long MWCNT bundles and performed tensile tests. The $E$ computed in the current work is comparable with the literature data presented in Table 12. The modulus of MWCNT bundle identified here is significantly higher as compared to that of SWCNT fibre system presented by Dalton et al. [39]. Dalton et al. [39] produced a SWCNT fibre system by spinning tubes within an epoxy resin and determined the modulus to be $80 \mathrm{GPa}$. The buckypaper modelled in Sect. 3.5 is assumed to be composed of MWCNT bundles with seven tubes. Therefore, the $E$ of MWCNT bundles considered in the subsequent buckypaper analysis is $1461.0 \mathrm{GPa}$. It can be noted that the process of irradiation [105] can introduce covalent bonds between the tubes within the bundle and also between the bundles of a buckypaper. As a result, the modulus of the bundle can be enhanced further [1].

The shear modulus $(G)$ of MWCNT bundles has been calculated by introducing shearing boundary conditions similar to the calculation of SWCNT (refer to Sect. 3.2) and using Eq. 12. All four configurations of MWCNT bundles shown in Fig. 1 have been analyzed under shear loading. The $G$ for configuration(2), configuration(3), configuration(5), and configuration(7) of MWCNTs are found to be 652.0, 664.0, 671.0, and $675.0 \mathrm{GPa}$, respectively. This indicates a marginal increase in shear stiffness with the addition of tubes. The variation of $G$ with respect to the number of tubes in the bundle is shown in Fig. 7c. The slope of the curve is not significant in this plot. No literature data exist that has analyzed the current configurations of MWCNT bundles under shear. However, Liu et al. [92] analyzed a bundle of seven SWCNTs and presented the value of $G$ as $7.0 \mathrm{GPa}$, where the shear loading on the SWCNT bundle was significantly different compared to that of the current work. The buckypaper modelled in Sect. 3.5 is assumed to be composed of MWCNT bundles with seven tubes. Therefore, the $G$ of MWCNT bundles considered in the subsequent buckypaper analysis is $675.0 \mathrm{GPa}$.

Poisson's ratio has been measured by introducing tensile forces to a MWCNT bundle, followed by the recording of transverse and axial strains. The $v$ has been computed for all four configurations of MWCNT bundles shown in Fig. 1. The Poisson's ratio as a function of the number of MWCNTs in a bundle is shown in Fig. 7d. The $v$ for configuration(2), configuration(3), configuration(5), and configuration(7) of MWCNTs are found to be $0.33,0.34,0.345$, and 0.35 , respectively. This shows a minimal increase in $v$ with the addition of MWCNTs into the bundle. No literature that determines Poisson's ratio of MWCNT bundle has been identified except that of Miao et al. [109], which used a CNT forest to dry spin the bundles into yarn and concluded a Poisson's ratio as high as 8.0. Such an unusually high value is the result of twisted configurations and porosity in the yarns. The buckypaper modelled in Sect. 3.5 is assumed to be composed of MWCNT bundles with seven tubes. Therefore, the $v$ of MWCNT bundles considered in the subsequent buckypaper analysis is 0.35 .

\subsection{Mechanical properties of MWCNT buckypaper composite}

The longitudinal and lateral moduli, denoted here as $E_{1}$ and $E_{2}$, respectively, have been computed for five variations of buckypaper composite. The five variants are based on stretched compositions of $0,20,40,60$, and $80 \%$, as shown in Fig. 3a-e. A cross-sectional view of the mesh with $100 \%$ wavy nanoropes, taken at the midplane, is shown in Fig. 3f. The finite-element-based modelling strategy of this buckypaper composite has been explained in Sect. 2.5. The variation of both longitudinal and transverse modulus with respect to the stretch concentration of buckypaper composites is shown in Fig. 8a. The longitudinal modulus is measured along the length of stretched nanoropes (i.e., CNT bundles), while the transverse modulus is measured perpendicular to the length of stretched nanoropes. In Fig. 8a, the presence of $20 \%$ stretched nanoropes leads to a longitudinal modulus of $80 \mathrm{GPa}$. This is higher than the Young's modulus offered by engineering aluminium. Importantly, the presence of $80 \%$ stretched nanoropes would lead to a longitudinal modulus of $370 \mathrm{GPa}$. Such a value of modulus is higher than that of engineering steel and carbon fibre. Figure 8a shows a buckypaper composite consisting of only un-stretched (wavy) nanoropes, where the observed longitudinal modulus is $45 \mathrm{GPa}$. It indicates that, by stretching nanoropes, an enhancement of modulus up-to eightfold can be achieved. Such a phenomenon can be attributed to the fibre length and orientation efficiency factors described in classical composite theories [78]. A comprehensive comparison against the literature concerning different classes of buckypaper is shown in Table 13. The modulus as per this table varies between 0.13 and $650.0 \mathrm{GPa}$, indicating a considerable scatter. However, the modulus of the current work $(80 \%$ stretched) is comparable to that of the other studies (Cheng et al. [35]), which also made use of the BMI as the resin in conjunction with an MWCNT buckypaper. This experimental work attained a modulus of $350.0 \mathrm{GPa}$ by functionalizing MWCNT paper with m-CPBA/dichloromethane solution and performing mechanical stretching. However, without functionalizing and mechanical stretching, the modulus of 


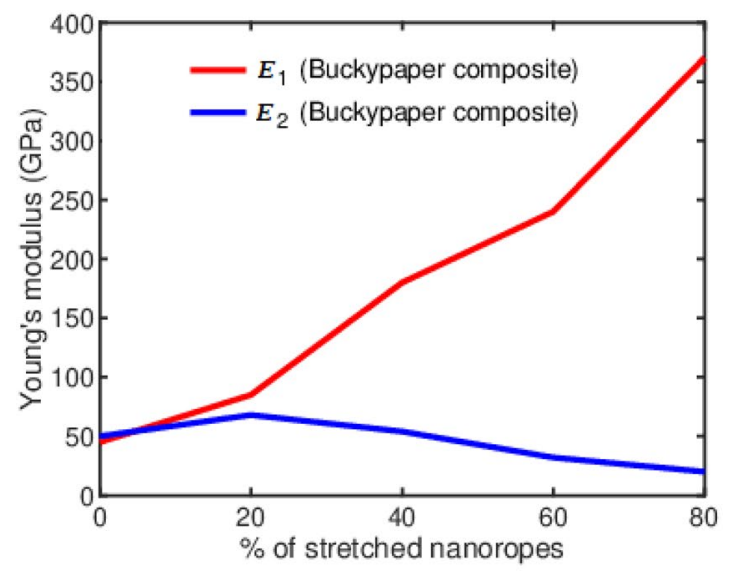

(a)

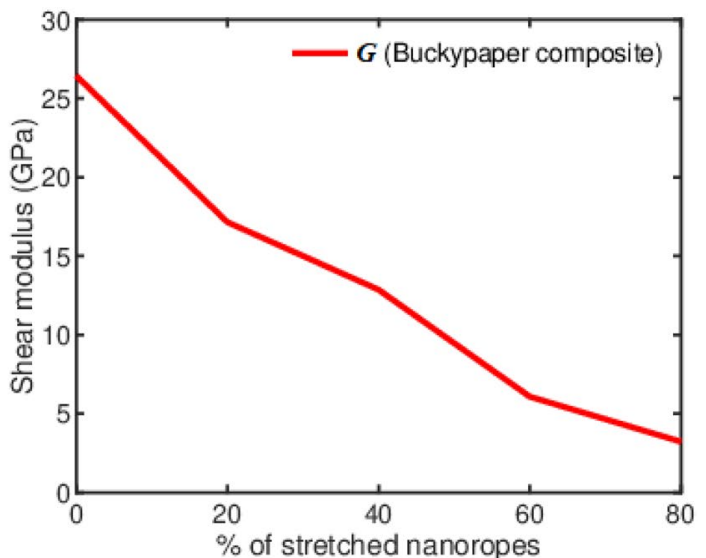

(b)

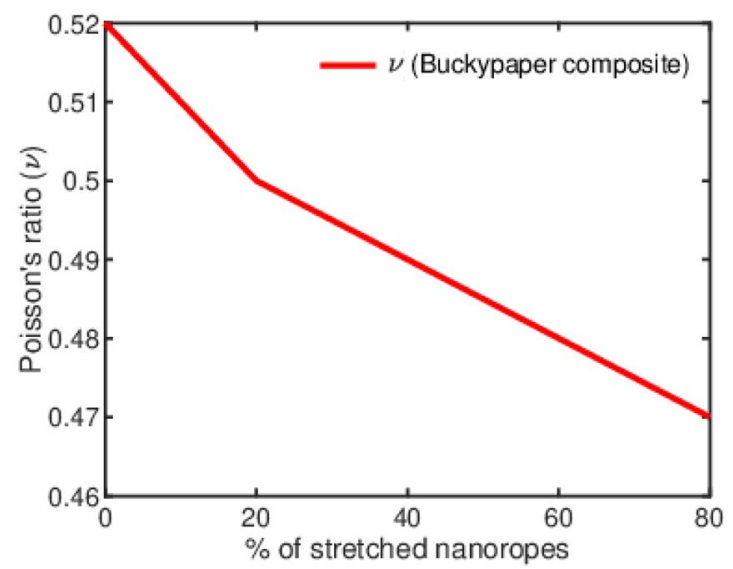

(c)

Fig. 8 Computation of mechanical properties of MWCNT buckypaper composite sheet. a Influence of stretched nanoropes on Young's modulus. b Influence of stretched nanoropes on in-plane shear modulus. c Influence of stretched nanoropes on the Poisson's ratio

composite paper was found to be $40.0 \mathrm{GPa}$ in [35], which is comparable with the current work (un-stretched).

Composite sheet shear modulus has been computed by constraining one edge and then introducing a shearing load along the length of the opposite edge. This simulation replicates a test sheet behaviour of ASTM D7078. The shear modulus has been computed for sheets with five stretch compositions of $0,20,40,60$, and $80 \%$, as shown in Fig. 8 b. The presence of wavy nanoropes offers higher in-plane shear modulus compared to that of stretched nanoropes in either of the two plane axes. Therefore, the buckypaper composite with $0 \%$ stretched nanoropes offers the highest in-plane shear modulus (27 GPa). As the stretch composition is increased, the shear modulus drops significantly and reaches as low ad $4 \mathrm{GPa}$ for the buckypaper composite with $80 \%$ stretched and $20 \%$ wavy nanoropes. This value of shear modulus will be used to further simulate buckypaper composite in the automotive suspension control arm presented in Sect. 3.6.
It may be noted that there are no literature data available on the shear modulus of buckypaper composites.

Poisson's ratio of the buckypaper composite has been measured by applying tensile force at one edge and constraining the opposite edge. The computed Poisson's ratio of buckypaper composite has been plotted in Fig. 8c. This plot shows a variation of Poisson's ratio $(v)$ with respect to the $\%$ of stretched nanoropes. Note that the stretched nanoropes are the fibres that are aligned along the direction of loading. As the content of stretched nanoropes is increased, stiffness along the stretch direction increases. This leads to a drop in Poisson's ratio, as noticeable in Fig. 8c. At $0 \%$ stretched nanoropes' content, the Poisson's ratio is 0.52 . The value of the Poisson's ratio with $80 \%$ stretched nanorope content is 0.47 . Thus, a drop of $9.6 \%$ in Poisson's ratio has been observed due to an enhancement in the alignment of nanoropes by $80 \%$. The Poisson's ratio determined in the current work has been compared against that of literature data in Table 14. The literature is 
Table 13 Young's modulus $(E)$ of graphene and CNT-based papers along with comparative results from existing literature

\begin{tabular}{|c|c|c|c|}
\hline Paper type/matrix type & $E(\mathrm{GPa})$ & Measurement method & Source \\
\hline Graphene oxide/polyvinyl alcohol & 26.0 & MD & {$[38]$} \\
\hline SWCNT/polyphenylene & 164.0 & Experimental & {$[90]$} \\
\hline SWCNT/polycarbonate & 5.1 & Experimental & [128] \\
\hline SWCNT/polyphenylene sulphide & 3.4 & Experimental & {$[42]$} \\
\hline SWCNT/polyether ether ketone & 4.8 & Experimental & {$[42]$} \\
\hline SWCNT/epoxy & 23.0 & FEM & {$[180]$} \\
\hline SWCNT/epoxy & 0.13 & Experimental & {$[180]$} \\
\hline SWCNT/polyvinyl pyrrolidone, & 6.9 & Experimental & {$[36]$} \\
\hline SWCNT/polyvinyl pyrrolidone & 3.2 & Experimental & [49] \\
\hline MWCNT/bismaleimide & 169.0 & Experimental & {$[34]$} \\
\hline MWCNT(functionalized and wavy)/bismaleimide & 40.0 & Experimental & {$[35]$} \\
\hline MWCNT(pristine and wavy)/bismaleimide & 150.0 & Experimental & {$[35]$} \\
\hline MWCNT(functionalized and stretched)/bismaleimide & 350.0 & Experimental & {$[35]$} \\
\hline MWCNT(pristine and stretched)/bismaleimide & 120.0 & Experimental & {$[35]$} \\
\hline SWCNT/silicone & 0.42 & Experimental & {$[83]$} \\
\hline SWCNT/polyether ether ketone & 3.9 & Experimental & {$[156]$} \\
\hline MWCNT/carrageenan & 2.7 & Experimental & {$[2]$} \\
\hline MWCNT/polyurethane & 6.2 & Experimental & {$[65]$} \\
\hline MWCNT/polyurethane & 8.3 & Mori-Tanaka & {$[65]$} \\
\hline MWCNT/epoxy prepreg & 50.1 & Experimental & {$[124]$} \\
\hline MWCNT/epoxy & 6.0 & Experimental & {$[62]$} \\
\hline MWCNT/parmax & 36.0 & Experimental & {$[30]$} \\
\hline MWCNT/polyimide & 40.0 & Experimental & {$[72]$} \\
\hline MWCNT/epoxy & 65.0 & Experimental & [118] \\
\hline SWCNT/polystyrene & 2.0 & Experimental & {$[20]$} \\
\hline Hybrid (MWCNT/carbon fibre/epoxy) & 177.0 & Experimental & {$[172]$} \\
\hline MWCNT/epoxy & 20.0 & Experimental & {$[172]$} \\
\hline Graphene nano platelet/polyetherimide & 22.5 & Experimental & {$[174]$} \\
\hline SWCNT/epoxy & 650.0 & Analytical & [187] \\
\hline MWCNT/cyclic butylene terephthalate & 110.0 & Experimental & {$[89]$} \\
\hline SWCNT/epoxy & 178.0 & MD & {$[122]$} \\
\hline MWCNT/polyvinyl alcohol & 1.3 & Experimental & {$[126]$} \\
\hline MWCNT/bismaleimide & 45.0 & FEM & Present (wavy) \\
\hline MWCNT/bismaleimide & 370.0 & FEM & Present (stretched) \\
\hline
\end{tabular}

available on a variety of buckypapers involving SWCNT or MWCNT, or graphene. The paper format of graphene is simply referred to as graphene paper in many articles. The Poisson's ratio of buckypaper composite is found to vary between 0.07 and 3.4 in the literature (Table 14). The Poisson's ratio determined in the current work falls well within this range. It is important to highlight that the reference [155] measured Poisson's ratio of MWCNT buckypaper/polyurethane composite under compressive loading. None of the articles in the literature is found to consider bismaleimide (BMI) as a resin, unlike the current work. The other research studies used different variants of the polymer as bulk materials in the composites to determine Poisson's ratio. The value of Poisson's ratio determined here will be used to further simulate the automotive suspension control arm in Sect. 3.6.

\subsubsection{Supplementary note: Strength of nanocomposites}

In this paper, the primary focus is on evaluating the effective elastic properties, leading to stiffness investigation at different levels of the length scales. However, the strength of raw materials also plays a vital role in the mechanical performance of an engineering component such as a control arm. A brief tensile analysis has been presented in this section to identify the value of strength buckypaper composites can offer to engineer components. The multi-scale modelling strategy used here is the same as our previous works [27-29]. The analysis is based on an idealized RVE, wherein 
Table 14 Poisson's ratio of graphene and CNT-based papers along with comparative results from existing literature

\begin{tabular}{llll}
\hline Base material & $v$ & Test method & Source \\
\hline MWCNT/polydimethylsiloxane & 0.07 & Experimental & {$[32]$} \\
SWCNT/epoxy & 0.48 & Experimental & {$[165]$} \\
SWCNT/epoxy & 0.51 & Numerical & {$[165]$} \\
SWCNT(long)/epoxy & 0.12 & Numerical & {$[122]$} \\
SWCNT(short)/epoxy & 0.17 & Numerical & {$[122]$} \\
MWCNT/polyvinyl alcohol & 0.5 & Experimental & {$[127]$} \\
MWCNT/graphene & 0.17 & Experimental & {$[126]$} \\
MWCNT/polyurethane & 0.5 & Experimental & {$[155]$} \\
SWCNT/polymer & 3.4 & Semi-numerical & {$[68]$} \\
MWCNT/epoxy & 0.4 & Assumed & {$[139]$} \\
SWCNT array/epoxy & 0.4 & Analytical & {$[6]$} \\
G/octadecylamine & 0.17 & Experimental & {$[136]$} \\
MWCNT/bismaleimide & 0.47 & Numerical & Present \\
\hline
\end{tabular}

CNTs are represented by traditional atomistic space frame models (described in Sect. 2.2) and the surrounding matrix is defined by 3D continuum finite elements. The multi-scale model development is shown in Fig. 9 and a comprehensive explanation about the methodology can be found in [29]. A high-fidelity RVE with CNT $60 \mathrm{vol} \%$ and BMI resin has been considered. A simplified geometry of buckypaper mat containing a pair of eight MWCNTs overlapped on another eight MWCNTs has been constructed (Fig. 9a). Inter-wall, intertube, and matrix tube interactions have been modelled by van der Waal's springs (Sect. 2.3). The composite RVE is shown in Fig. 9b. Nanoropes have not been considered here due to a lack of computational resources. The stress-strain non-linearity as per [111] has been defined for the $\mathrm{C}-\mathrm{C}$ bonds. Material non-linearity in the BMI resin has also been modelled using experimental stress-strain data [138]. The RVE has been clamped at one end, and a tensile deflection has been applied to the opposite end. The reaction force measured at the clamped end has been used to calculate stress. The deformation in the RVE has been used to compute strain. The resulting stress-strain curve is shown in Fig. 9d. The curve is found to be linear up to $78 \mathrm{GPa}$ of stress and $7 \%$ of strain. The curve takes a non-linear diversion beyond this stress due to various mechanisms such as non-linearity in resin and CC bonds, and debonding of

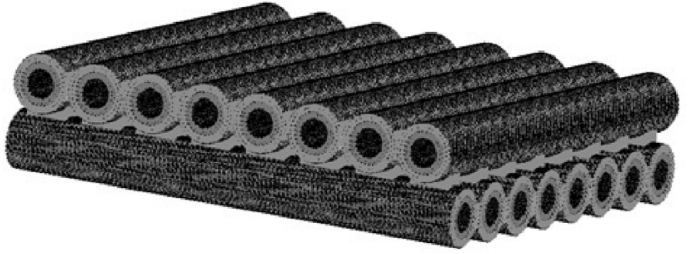

(a) Idealized section of a buckypaper

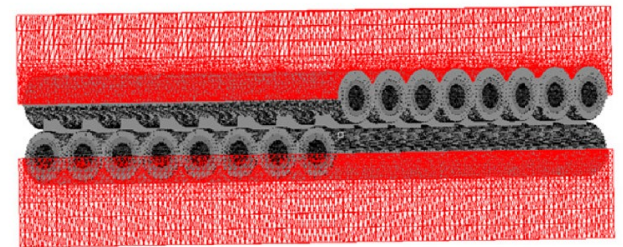

(b) RVE of a buckypaper composite

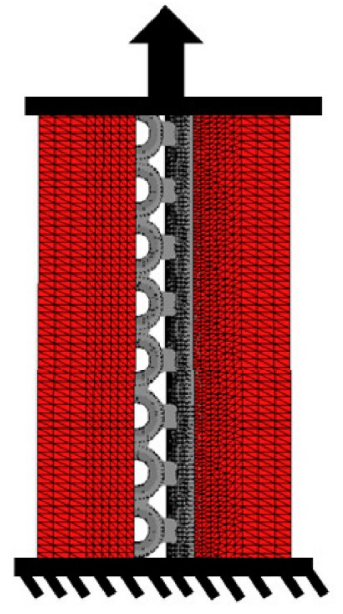

(c) Prescribed tensile boundary conditions on the RVE

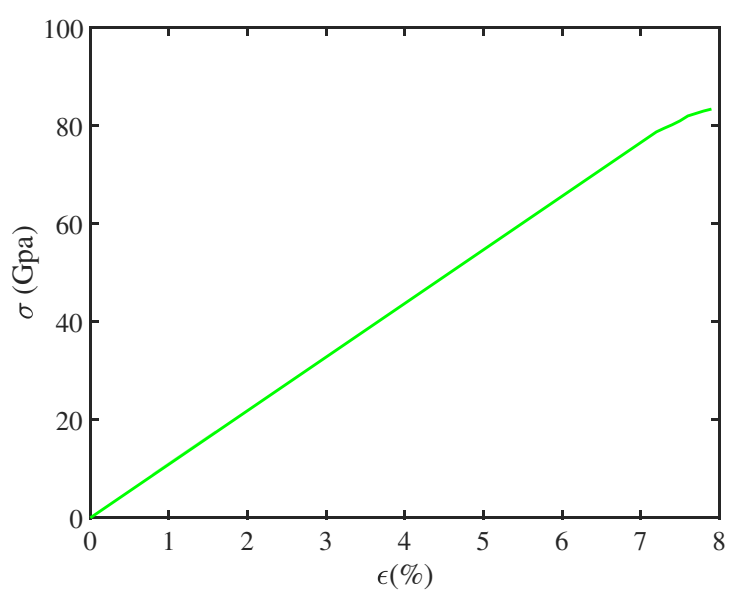

(d) Tensile stress-strain curve

Fig. 9 Multi-scale simulation of buckypaper composites, leading to the stress-strain curve (Note: the volume of matrix shown here is scaled for visual clarity.) 
LJ springs [29]. The analysis beyond $8 \%$ of strain has not been continued due to computational restrictions. The current stress-strain behaviour of the idealized RVE suggests that the strength of the nanocomposite can be at least as high as $78 \mathrm{GPa}$. Such a tensile strength is far beyond what conventional engineering materials can offer. The current stress-strain behaviour is dominated by the CNT's response to tension as a result of simplified boundary conditions. The other factors such as higher CNT fraction (60 vol\%) and tube alignments along the direction of loads are also influencing the strength of RVE. In practice, the boundary conditions and tube alignments can be different from those in the current RVE and it should be modelled accordingly.

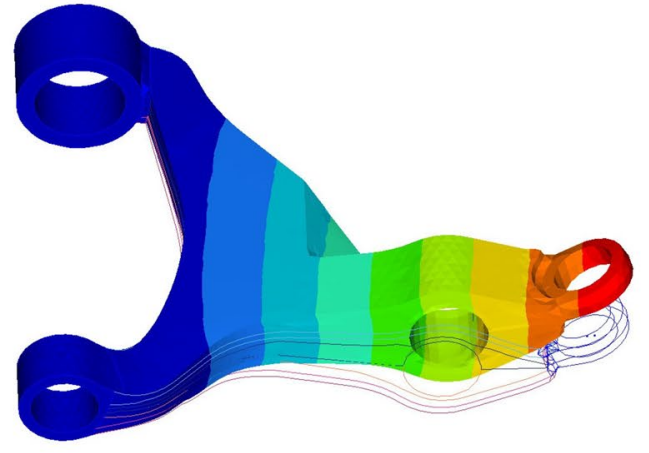

(a) Mode 1

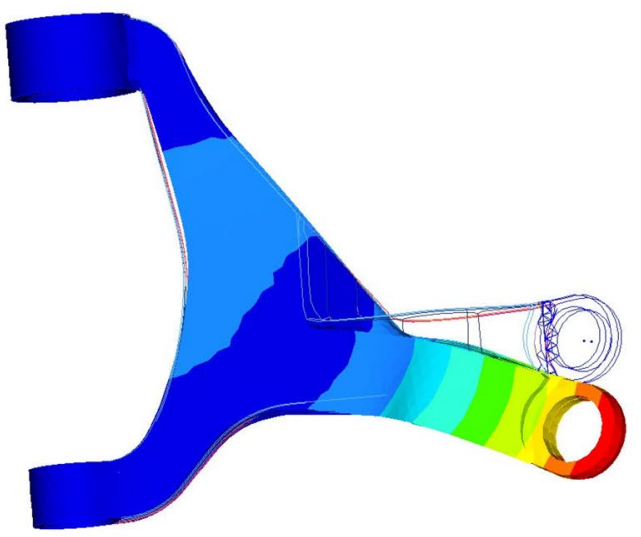

(c) Mode 3

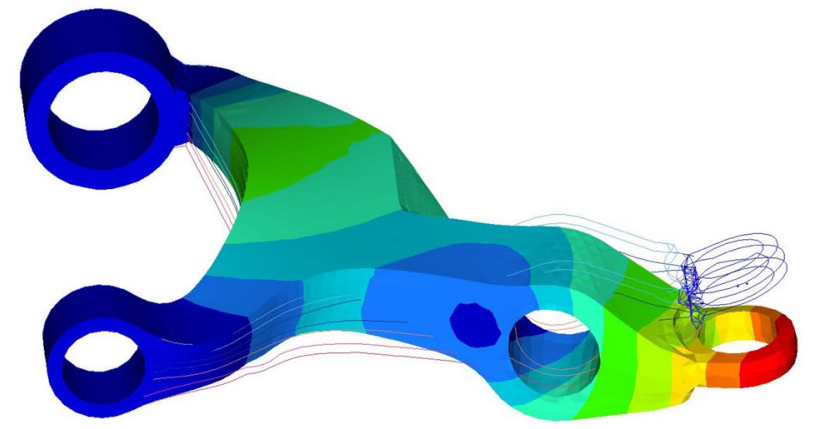

(b) Mode 2

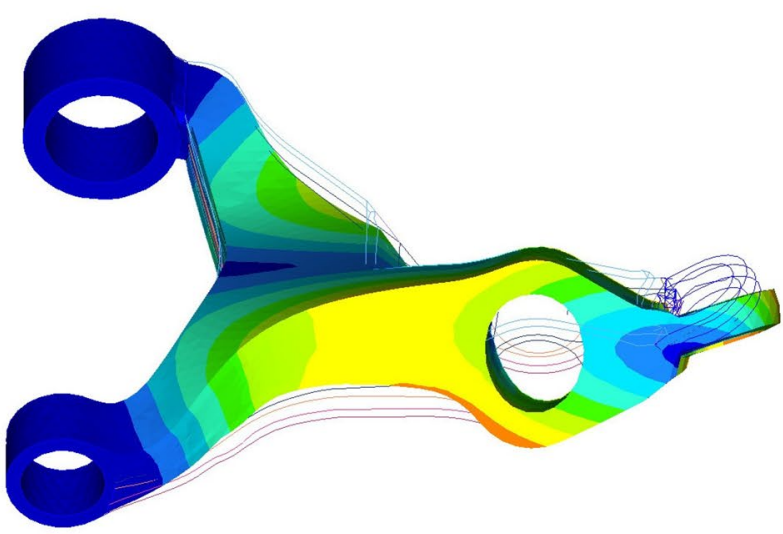

(d) Mode 4

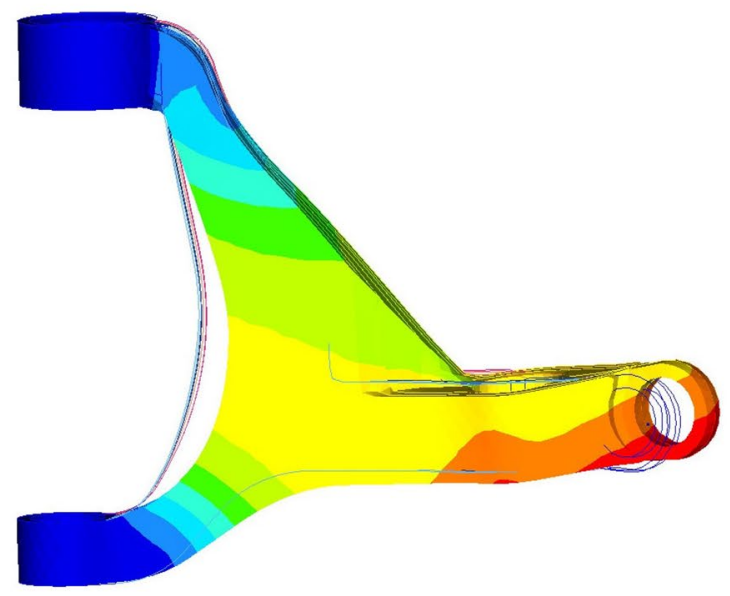

(e) Mode 5

Fig. 10 First five mode shapes of the conceptual composite control arm. The corresponding natural frequencies are presented in Table 15 


\subsection{Analysis of an automotive control arm}

We first investigate the dynamic behaviour of the composite control arm. The first five vibration mode shapes of the control arm are shown in Fig. 10. The first mode shape (refer to Fig. 10a) is of vertical cantilever bending behaviour that simulates vertical tire motion along the McPherson strut, the second mode shape (refer to Fig. 10b) is an in-plane bending type that simulates wheel acceleration, the third mode shape (refer to Fig. 10c) is a coupled downward vertical motion and in-plane bending, the fourth mode shape (refer to Fig. 10d) is torsional with respect to wheel axis, and the fifth mode shape (refer to Fig. 10e) is an inboard horizontal wheel traversing type. These five mode shapes are found to be similar to the baseline design. It is because the geometry of the design has not been significantly altered while redesigning the baseline into an alternative lightweight conceptual design. An effort has been made to replace materials within the available baseline design volume of the control arm. Furthermore, the distance and the angle between the hardpoints also influence the mode shape. Since the location of hardpoints has not been altered while redesigning the baseline into a conceptual design, the mode shapes are expected to remain the same. However, the natural frequencies become significantly different when the baseline design is compared against the new composite design. Since the mode shapes are similar, it is possible to compare the baseline natural frequencies against the new concept design at a given mode shape.

Here, the natural frequencies of the proposed lightweight concept design will be bench-marked against the baseline design. All the natural frequencies in this section are presented in cycles per second, also referred to as Hertz $(\mathrm{Hz})$. The natural frequencies are mainly dependent on the stiffness and mass matrices of the component. The higher the stiffness of the component, higher will be the natural frequency. Furthermore, the lower the mass, higher will be natural frequency. This means that light-weighting the control arm from baseline aluminium to a composite equivalent will enhance the natural frequency. Also, by enhancing the control arm's stiffness by replacing low modulus aluminium material with high-modulus materials such as buckypaper and carbon fibre prepreg will enhance the natural frequency of the control arm, since the stiffness of any material is derived based on its modulus.

It is important to ensure the orientation of carbon nanotubes and carbon fibres in buckypaper and prepreg with respect to mode shapes to witness natural frequency enhancements. To influence the natural frequencies of all mode shapes, the orientations of buckypaper and prepreg plies have been kept as $0^{\circ}, 45^{\circ}, 60^{\circ}$, and $90^{\circ}$. This ply arrangement offers a certain degree of isotropy (known as in-plane isotropy) to the laminates in the conceptual control arm. The first five natural frequencies of the baseline design and the proposed concept design are shown in Table 15 . These natural frequencies are relevant to the mode shapes shown in Fig. 10. The baseline design's natural frequency varies from 365.0 to $3045.0 \mathrm{~Hz}$, for the first five mode shapes. The conceptual control arm's natural frequency varies from 440.0 to $3801.0 \mathrm{~Hz}$, for the first five mode shapes. It is important to ensure that the mode-specific natural frequencies of the chassis body and other components attached to the control arm do not coincide with these frequencies to avoid resonance. The numerical results reveal that the conceptual control arm over-performs as compared to the baseline design. For instance, the first and fifth natural frequencies are enhanced by 21 and $25 \%$, respectively, for the concept design compared to the baseline design. Weight of the control arm drops from 4.2 to $1.8 \mathrm{~kg}$ due to light-weighting. This accounts for $57 \%$ of weight savings, leading to a considerable reduction in vehicle energy consumption. With the aid of evolving virtual intelligence tools such as topology and ply stacking optimisation, the mechanical performance can be further enhanced along with reduction of weight.

Besides the dynamic characterization, performance of a component under static loading is equally crucial to come up with a successful design. Here, we compare the performance of the lightweight/buckypaper-reinforced control arm against the metallic counterpart, under tensile, compressive, shear, and bending loads. The finite-element method and model implemented here are discussed in Sect. 3.6. A load of $10 \mathrm{kN}$ has been defined on hardpoint 4 to simulate tension, compression, shearing, and bending in four separate load cases. The control arm has been fully constrained at hardpoints 1 and 2 . The definition of hardpoints can be seen in Fig. 4. The resulting deflections
Table 15 Static deflections (measured in $\mathrm{mm}$ ) and first five natural frequencies (measured in $\mathrm{Hz}$ ) of the baseline design and the proposed composite design for automotive control arm

\begin{tabular}{|c|c|c|c|c|c|}
\hline \multicolumn{3}{|l|}{ Static analysis } & \multicolumn{3}{|c|}{ Dynamic analysis } \\
\hline & Baseline & Concept design & & Baseline & Concept design \\
\hline Tension & 0.015 & 0.010 & Mode 1 & 365 & 440 \\
\hline Compression & 0.015 & 0.013 & Mode 2 & 1511 & 1822 \\
\hline Shear & 0.013 & 0.008 & Mode 3 & 1726 & 2085 \\
\hline \multirow[t]{2}{*}{ Bending } & 0.014 & 0.010 & Mode 4 & 2933 & 3588 \\
\hline & & & Mode 5 & 3045 & 3801 \\
\hline
\end{tabular}




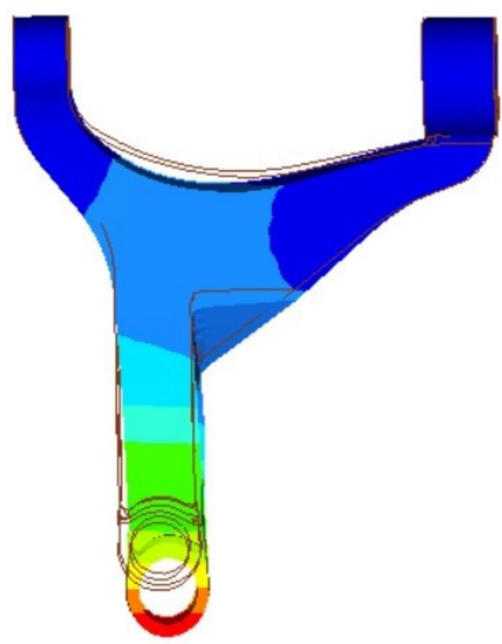

(a) Tensile deflection

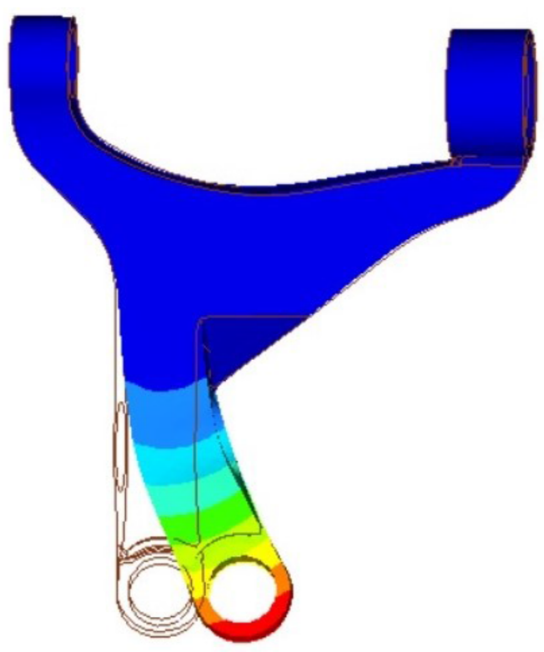

(c) Shear deflection

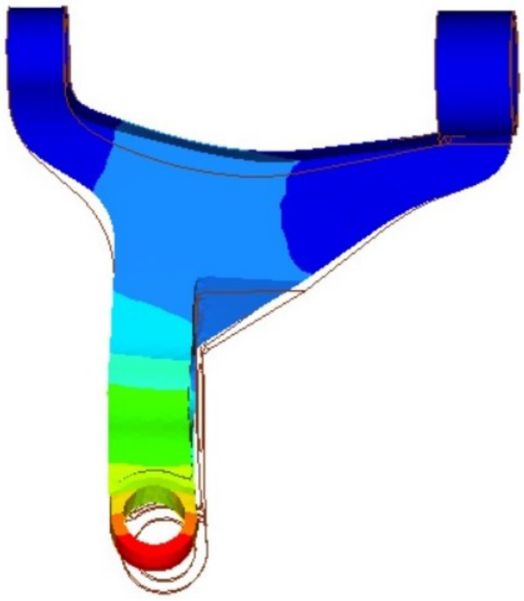

(b) Compressive deflection

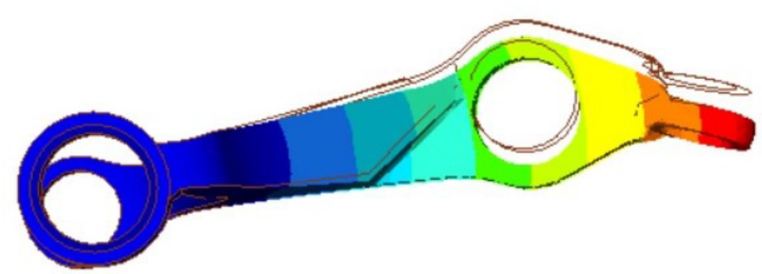

(d) Bending deflection

Fig. 11 Static deformed shapes of the control arm under four different types of loading. Note that the force is applied at hard point 4 (refer to Fig. 4) keeping the hard points 1 and 2 constrained

in the conceptual control arm are shown in Fig. 11. In the tensile test, the tip of the control arm (hardpoint 4) has been pulled in the horizontal direction with the aid of $10 \mathrm{kN}$ force. In the compression test, the tip of the control arm (hardpoint 4) has been pushed in the horizontal direction with the aid of $10 \mathrm{kN}$ force. In the shear test, the tip of the control arm (hardpoint 4) has been displaced sideways with the aid of $10 \mathrm{kN}$ force. In the bending test, the tip of the control arm (hardpoint 4) has been pushed downwards with the aid of $10 \mathrm{kN}$ force. The resulting tensile, compressive, shearing, and bending deflections can be seen in Fig. 11a-d respectively. The total deflections recorded from the finite-element analysis are tabulated in Table 15.
This table compares the performance of the conceptual buckypaper-based control arm against that of the baseline design. These results measure the tensile, compressive, shearing, and bending stiffness of aluminium and buckypaper-based composite materials within the control arm. The results conclude that the conceptual control arm outperforms the baseline design under all four load cases. The enhancements in tensile, compressive, shearing, and bending stiffness are found to be $34,13,37$, and $28 \%$. In summary, the numerical results concerning both dynamic and static analyses convincingly demonstrate that the proposed buckypaper reinforced composite control arm 
provides enhanced mechanical performance while achieving a significant reduction of weight.

\section{Conclusions}

This article develops a computational bridging of different length scales involving six levels in the range of nano- to macro-scale behaviour of buckypaper composites and the structural components manufactured thereof. The sequential derivatives of carbon at six levels are analyzed involving graphene, CNT, CNT bundle, buckypaper, and buckypaper composites. We have developed a coupled atomistic-continuum modelling approach for the multi-level simulations. Graphene, CNTs, and CNT bundles are modelled using atomistic simulations, while the buckypaper and its composites are modelled using equivalent beam representations for the bundles and continuum solid representation for resin. At the atomic level, we start by establishing the mechanical equivalence of $\mathrm{C}-\mathrm{C}$ bonds as idealized beams, followed by quantification of the equivalent elastic properties of CNTs and CNT bundles based on a nanoscale finite-element analysis. At the next level, we characterize the mechanical properties of buckypapers and buckypaper composites as programmed networks of CNTs and CNT bundles, which are idealized as equivalent beams with effective properties computed at the preceding length scale level. The simulation results at each level are extensively validated and bench-marked with various configurations considering the data available in the literature. The most salient points concerning the current investigation are listed below

- This research paper is the first of its kind to present an automotive structural concept design that uses buckypaper composites as one of the two primary reinforcements. More importantly, the investigation starts at a fundamental nanoscale level and the critical static and dynamic behaviour are characterized at the macro-scale structural level through seamless multi-scale propagation of effective mechanical information.

- This article comprehensively captures various levels of effective mechanical behaviour necessary to characterize the macroscopic structural responses. The total number of levels considered here is six, which are bridged through a coupled atomistic-continuum simulation strategy.

- The level 1 simulation focuses on characterizing the Young's modulus, shear modulus, and Poisson's ratio of single-layer graphene sheets through an atomistic finiteelement framework. It is noted that for a single-layer graphene sheet, the Young's modulus can be as high as 1082.0 GPa, and the shear modulus can be as high as 606.0 GPa, while the Poisson's ratio comes out to be 0.62 . Such results obtained through the current computationally efficient framework agree well with the experi- mental and molecular dynamics studies presented in the literature.

- The level 2 simulation explores Young's modulus, shear modulus, and Poisson's ratio of single-walled carbon nanotubes through the atomistic finite-element framework. At this level, Young's modulus can be as high as 1080.0 GPa, and the shear modulus can be as high as 504.0 GPa, while the Poisson's ratio is found to be 0.37 .

- The level 3 simulation computes Young's modulus, shear modulus, and Poisson's ratio of multi-walled carbon nanotubes considering multiple configurations. At this level, the Young's modulus can be as high as $1401.0 \mathrm{GPa}$, and the shear modulus can be as high as $638.0 \mathrm{GPa}$, while the Poisson's ratio remains to be 0.32 .

- The level 4 simulation computes Young's modulus, shear modulus, and Poisson's ratio of multi-walled carbon nanotube bundles (also known as nanoropes) considering multiple configurations, some of which have hitherto been unexplored. At this level, Young's modulus can be as high as $1461.0 \mathrm{GPa}$, the shear modulus can be as high as $675.0 \mathrm{GPa}$, while the Poisson's ratio is noted to be around 0.47 .

- The level 5 simulation focuses on Young's modulus, shear modulus, and Poisson's ratio of buckypaper composites involving carbon nanotubes and bismaleimide resin. The equivalent properties obtained in the preceding levels are exploited at this level for carrying out a reduced order analysis without compromising the accuracy. Such an efficient analysis framework led to the feasibility of characterizing a wide range of configurations. For buckypaper composites, the Young's modulus can be as high as $370.0 \mathrm{GPa}$, and the shear modulus can be as high as $27.0 \mathrm{GPa}$, while the Poisson's ratio comes out to be 0.62 .

- In the level 6 of simulation, we focus on the full-scale structural analysis of a concept control arm made of buckypaper reinforced composites. Both the dynamic and static performances are compared with traditional monolithic metallic designs based on a finite-element analysis using the effective mechanical properties of buckypaper obtained in level 5. The numerical results demonstrate that a buckypaper composite can enhance the natural frequency and stiffness up to 25 and $37 \%$ with respect to conventional monolithic metallic designs, while reducing the weight by $57 \%$.

- With the aid of evolving virtual intelligence tools such as topology and ply-stacking optimisation, the mechanical performance of buckypaper composites can be further enhanced along with reduction of weight. Moreover, we note that there is an immense scope of designing high-performance composites according to the application-specific demands of tailored anisotropy through the expanded design space of single- and 
multi-walled nanotube bundles with multiple possible configurations, their shape, relative density, and orientation.

The current article presents an efficient atomistic finite-element-based modelling framework, wherein the contribution is twofold: I. Insightful new results unravelling the mechanical behaviour, II. Development of the generic atomistic finite-element framework for efficient mechanical analysis involving multiple length scales. Adoption of the atomistic finite-element approach instead of conventional simulation methods such as MD simulation brings significantly more computational efficiency in the analysis and the proposed approach is a panacea in situations where inter-atomic potentials required for carrying out MD simulations are unavailable in case of many complex systems.

In summary, the numerical outcomes suggest that carbonbased nanostructural derivative in the form of buckypaper can significantly improve the mechanical properties of advanced lightweight structural components as reinforcements for the next generation of aerospace and automotive structures. The characterization of elastic properties at different levels of the derivatives of carbon and their composites along with the proposed multi-scale framework of atomistic-continuum simulations will crucially support further developments in this field.

Supplementary Information The online version contains supplementary material available at https://doi.org/10.1007/s00366-021-01538-w.

Acknowledgements SA acknowledges the European Commission grant under Marie Skłodowska Curie Actions (Grant number 799201-METACTIVE). TM would like to acknowledge the initiation grant received from IIT Kanpur during the research work.

Open Access This article is licensed under a Creative Commons Attribution 4.0 International License, which permits use, sharing, adaptation, distribution and reproduction in any medium or format, as long as you give appropriate credit to the original author(s) and the source, provide a link to the Creative Commons licence, and indicate if changes were made. The images or other third party material in this article are included in the article's Creative Commons licence, unless indicated otherwise in a credit line to the material. If material is not included in the article's Creative Commons licence and your intended use is not permitted by statutory regulation or exceeds the permitted use, you will need to obtain permission directly from the copyright holder. To view a copy of this licence, visit http://creativecommons.org/licenses/by/4.0/.

\section{References}

1. Åström JA, Krasheninnikov AV, Nordlund K (2004) Carbon nanotube mats and fibers with irradiation-improved mechanical characteristics: a theoretical model. Phys Rev Lett 93:215503

2. Aldalbahi A, in het Panhuis M (2012) Electrical and mechanical characteristics of buckypapers and evaporative cast films prepared using single and multi-walled carbon nanotubes and the biopolymer carrageenan. Carbon 50(3):1197-1208

3. Altair Engineering Inc. OPTISTRUCT 11. Documentation and user manual. Accessed 21 Sept 2021

4. Alzebdeh K (2012) Evaluation of the in-plane effective elastic moduli of single-layered graphene sheet. Int J Mech Mater Des 8:269-278

5. Alzebdeh K (2014) An atomistic-based continuum approach for calculation of elastic properties of single-layered graphene sheet. Solid State Commun 177:25-28

6. Ashrafi B, Hubert P (2006) Modeling the elastic properties of carbon nanotube array/polymer composites. Compos Sci Technol 66(3):387-396

7. Askari D, Ghasemi-Nejhad MN (2011) Effects of vacancy defects on mechanical properties of graphene/carbon nanotubes: a numerical modeling. J Comput Theor Nanosci 8(4):783-794

8. Avila AF, Eduardo AC, Neto AC (2011) Vibrational analysis of graphene based nanostructures. Comput Struct 89(11):878-892. Computational Fluid and Solid Mechanics 2011

9. Avila AF, Lacerda GSR (2008) Molecular mechanics applied to single-walled carbon nanotubes. Mat Res 11(3):325-333

10. Ayatollahi M, Shadlou S, Shokrieh M (2011) Multiscale modeling for mechanical properties of carbon nanotube reinforced nanocomposites subjected to different types of loading. Compos Struct 93(9):2250-2259

11. Bai Y, Zhang R, Ye X, Zhu Z, Xie H, Shen B, Cai D, Liu B, Zhang C, Jia Z, Zhang S, Li X, Wei F (2018) Carbon nanotube bundles with tensile strength over $80 \mathrm{GPa}$. Nat Nanotechnol 13(7):589-595

12. Banhart F (2001) The formation of a connection between carbon nanotubes in an electron beam. Nano Lett 1(6):329-332

13. Baughman RH, Cui C, Zakhidov AA, Iqbal Z, Barisci JN, Spinks GM, Wallace GG, Mazzoldi A, De Rossi D, Rinzler AG, Jaschinski O, Roth S, Kertesz M (1999) Carbon nanotube actuators. Science 284(5418):1340-1344

14. Baykasoglu C, Mugan A (2012) Coupled molecular continuum mechanical modeling of graphene sheets. Physica E 45:151-161

15. Berhan L, Yi YB, Sastry AM (2004) Effect of nanorope waviness on the effective moduli of nanotube sheets. J Appl Phys 95(9):5027-5034

16. Berhan L, Yi YB, Sastry AM, Munoz E, Selvidge M, Baughman $R$ (2004) Mechanical properties of nanotube sheets: alterations in joint morphology and achievable moduli in manufacturable materials. J Appl Phys 95(8):4335-4345

17. Bradshaw R, Fisher F, Brinson L (2003) Fiber waviness in nanotube-reinforced polymer composites-II: modeling via numerical approximation of the dilute strain concentration tensor. Compos Sci Technol 63(11):1705-1722. Modeling and characterization of nanostructured materials

18. Brcic M, Canadija M, Brnic J, Lanc D, Krscanski S, Vukelic G (2009) Fe modelling of multi-walled carbon nanotubes. Estonian J Eng 15(2):77-86

19. Cha J, Jun GH, Park JK, Kim JC, Ryu HJ, Hong SH (2017) Improvement of modulus, strength and fracture toughness of CNT/epoxy nanocomposites through the functionalization of carbon nanotubes. Compos Part B Eng 129:169-179

20. Chadwick RC, Khan U, Coleman JN, Adronov A (2013) Polymer grafting to single-walled carbon nanotubes: effect of chain length on solubility, graft density and mechanical properties of macroscopic structures. Small 9(4):552-560

21. Chandra Y, Adhikari S, Saavedra Flores E, Figiel Ł (2020) Advances in finite element modelling of graphene and associated nanostructures. Mater Sci Eng R Rep 140:100544

22. Chandra Y, Chowdhury R, Adhikari S, Scarpa F (2011) Elastic instability of bilayer graphene using atomistic finite element. Phys E Low Dimens Syst Nanostruct 44(1):12-16 
23. Chandra Y, Chowdhury R, Scarpa F, Adhikaricor S (2011) Vibrational characteristics of bilayer graphene sheets. Thin Solid Films 519(18):6026-6032

24. Chandra Y, Chowdhury R, Scarpac F, Adhikarib S, Sienza J, Arnoldd C, Murmu T, Boulda D (2012) Vibration frequency of graphene based composites: a multiscale approach. Mater Sci Eng B 177:303-310

25. Chandra Y, Mukhopadhyay T, Adhikari S, Figiel Ł (2020) Sizedependent dynamic characteristics of graphene based multi-layer nano hetero-structures. Nanotechnology 31(14):145705

26. Chandra Y, Saavedra Flores E, Adhikari S (2020) Buckling of 2D nano hetero-structures with Moire patterns. Comput Mater Sci 177:109507

27. Chandra Y, Saavedra Flores EI, Scarpa F, Adhikari S (2016) Buckling of hybrid nano composites with embedded graphene and carbon nanotubes. Physica E 83:434-441

28. Chandra Y, Scarpa F, Adhikari S, Zhang J, Saavedra Flores EI, Peng H (2016) Pullout strength of graphene and carbon nanotube/epoxy composites. Compos Part B 10:1-8

29. Chandra Y, Scarpa F, Chowdhury R, Adhikari S, Sienz J (2013) Multiscale hybrid atomistic-FE approach for the nonlinear tensile behaviour of graphene nanocomposites. Sci Direct 46:147-153

30. Chang C-Y, Phillips EM, Liang R, Tozer SW, Wang B, Zhang C, Chiu H-T (2012) Alignment and properties of carbon nanotube buckypaper/liquid crystalline polymer composites. J Appl Polym Sci 128(3):1360-1368

31. Chang T, Gao H (2003) Size-dependent elastic properties of a single-walled carbon nanotube via a molecular mechanics model. J Mech Phys Solids 51(6):1059-1074

32. Chen L, Liu C, Wang J, Zhang W, Hu C, Fan S (2009) Auxetic materials with large negative Poisson's ratios based on highly oriented carbon nanotube structures. Appl Phys Lett 94(25):253111

33. Cheng H-C, Liu Y-L, Hsu Y-C, Chen W-H (2009) Atomisticcontinuum modeling for mechanical properties of single-walled carbon nanotubes. Int J Solids Struct 46(7):1695-1704

34. Cheng Q, Bao J, Park J, Liang Z, Zhang C, Wang B (2009) High mechanical performance composite conductor: multi-walled carbon nanotube sheet/bismaleimide nanocomposites. Adv Funct Mater 19(20):3219-3225

35. Cheng Q, Wang B, Zhang C, Liang Z (2010) Functionalized carbon-nanotube sheet/bismaleimide nanocomposites: mechanical and electrical performance beyond carbon-fiber composites. Small 6(6):763-767

36. Coleman JN, Blau WJ, Dalton AB, Muñoz E, Collins S, Kim BG, Razal J, Selvidge M, Vieiro G, Baughman RH (2003) Improving the mechanical properties of single-walled carbon nanotube sheets by intercalation of polymeric adhesives. Appl Phys Lett 82(11):1682-1684

37. Coluci VR, Hall LJ, Kozlov ME, Zhang M, Dantas SO, Galvão DS, Baughman RH (2008) Modeling the auxetic transition for carbon nanotube sheets. Phys. Rev. B 78:115408

38. Compton OC, Cranford SW, Putz KW, An Z, Brinson LC, Buehler MJ, Nguyen ST (2012) Tuning the mechanical properties of graphene oxide paper and its associated polymer nanocomposites by controlling cooperative intersheet hydrogen bonding. ACS Nano 6(3):2008-2019

39. Dalton AB, Collins S, Muñoz E, Razal JM, Ebron VH, Ferraris JP, Coleman JN, Kim BG, Baughman RH (2003) Super-tough carbon-nanotube fibres. Nature 423:703

40. Dassault Systemes-Simulia. ABAQUS version 6.14. Documentation and user manual. Accessed 21 Sep t 2021

41. Dey S, Mukhopadhyay T, Adhikari S (2018) Uncertainty quantification in laminated composites: a meta-model based approach. CRC Press, Boca Raton

42. Díez-Pascual AM, Guan J, Simard B, Gómez-Fatou MA (2012) Poly(phenylene sulphide) and poly(ether ether ketone) composites reinforced with single-walled carbon nanotube buckypaper: II-mechanical properties, electrical and thermal conductivity. Compos Part A Appl Sci Manuf 43(6):1007-1015

43. Domínguez-Rodríguez G, Tapia A, Avilés F (2014) An assessment of finite element analysis to predict the elastic modulus and Poisson's ratio of singlewall carbon nanotubes. Comput Mater Sci 82:257-263

44. Eslami Afrooz I, Öchsner A, Rahmandoust M (2012) Effects of the carbon nanotube distribution on the macroscopic stiffness of composite materials. Comput Mater Sci 51(1):422-429

45. Fan CW, Huang JH, Hwu CB, Liu YY (2008) Mechanical properties of single-walled carbon nanotubes-a finite element approach. Advances in fracture and materials behavior. Advanced materials research, vol 33. Trans Tech Publications, Zurich, pp 937-942

46. Fan CW, Liu YY, Hwu C (2009) Finite element simulation for estimating the mechanical properties of multi-walled carbon nanotubes. Appl Phys A 95(3):819-831

47. Feng Q-P, Shen X-J, Yang J-P, Fu S-Y, Mai Y-W, Friedrich K (2011) Synthesis of epoxy composites with high carbon nanotube loading and effects of tubular and wavy morphology on composite strength and modulus. Polymer 52(26):6037-6045

48. Fisher FT, Bradshaw RD, Brinson LC (2002) Effects of nanotube waviness on the modulus of nanotube-reinforced polymers. Appl Phys Lett 80(24):4647-4649

49. Frizzell CJ, in het Panhuis M, Coutinho DH, Balkus KJ, Minett AI, Blau WJ, Coleman JN (2005) Reinforcement of macroscopic carbon nanotube structures by polymer intercalation: the role of polymer molecular weight and chain conformation. Phys Rev B 72:245420

50. Fuhrer MS, Nygård J, Shih L, Forero M, Yoon Y-G, Mazzoni MSC, Choi HJ, Ihm J, Louie SG, Zettl A, McEuen PL (2000) Crossed nanotube junctions. Science 288(5465):494-497

51. Ge J, Jiang H, Sun ZY, Yu GJ, Su B, Sun T (2017) Calculating mechanics characteristics of single-walled carbon nanotube materials by finite element method. Innovative materials: engineering and applications II. Key engineering materials, vol 730. Trans Tech Publications, Zurich, pp 548-553

52. Gelin B (1994) Molecular modeling of polymer structures and properties. Hanser Gardner Publications, Cincinnati

53. Genoese A, Genoese A, Rizzi N, Salerno G (2016) On the derivation of the elastic properties of lattice nanostructures: the case of graphene sheets. Compos Part B Eng 115:316-329

54. Georgantzinos S, Giannopoulos G, Anifantis N (2010) Numerical investigation of elastic mechanical properties of graphene structures. Mater Des 31(10):4646-4654

55. Georgantzinos S, Katsareas D, Anifantis N (2011) Graphene characterization: a fully non-linear spring-based finite element prediction. Phys E Low Dimens Syst Nanostruct 43(10):1833-1839

56. Georgantzinos S, Katsareas D, Anifantis N (2012) Limit load analysis of graphene with pinhole defects: a nonlinear structural mechanics approach. Int J Mech Sci 55:85-94

57. Georgantzinosae S, Giannopoulosb G, Anifantisa N (2016) Coupled thermomechanical behavior of graphene using the spring-based finite element approach. J Appl Phys 120:014305

58. Ghavamian A, Rahmandoust M, Ochsner A (2013) On the determination of the shear modulus of carbon nanotubes. Compos Part B 44:52-59

59. Giannopoulos G, Kallivokas I (2014) Mechanical properties of graphene based nanocomposites incorporating a hybrid interphase. Finite Elem Anal Des 90:31-40

60. Girifalco LA, Hodak M, Lee RS (2000) Carbon nanotubes, buckyballs, ropes, and a universal graphitic potential. Phys Rev B 62:13104-13110

61. Gonnet P, Liang Z, Choi ES, Kadambala RS, Zhang C, Brooks JS, Wang B, Kramer L (2006) Thermal conductivity 
of magnetically aligned carbon nanotube buckypapers and nanocomposites. Curr Appl Phys 6(1):119-122

62. Guo J, Li M, Liu Q, Gu Y, Li Y, Zhang Z (2013) Influence of oxidation and distribution of carbon nanotube on mechanical properties of buckypaper/epoxy composites. J Reinf Plast Compos 32(4):248-257

63. Gupta KK, Mukhopadhyay T, Roy A, Dey S (2020) Probing the compound effect of spatially varying intrinsic defects and doping on mechanical properties of hybrid graphene monolayers. J Mater Sci Technol 50:44-58

64. Hall LJ, Coluci VR, Galvão DS, Kozlov ME, Zhang M, Dantas SO, Baughman RH (2008) Sign change of Poisson's ratio for carbon nanotube sheets. Science 320(5875):504-507

65. Han J-H, Zhang H, Chen M-J, Wang G-R, Zhang Z (2014) CNT buckypaper/thermoplastic polyurethane composites with enhanced stiffness, strength and toughness. Compos Sci Technol 103:63-71

66. Hemath M, Mavinkere Rangappa S, Kushvaha V, Dhakal HN, Siengchin S (2020) A comprehensive review on mechanical, electromagnetic radiation shielding, and thermal conductivity of fibers/inorganic fillers reinforced hybrid polymer composites. Polym Compos 41(10):3940-3965

67. Hexcel Composites. HexPly 8552. Datasheets. Accessed 01 Sep 2021

68. Tsai C, Zhang C, Jack DA, Liang R, Wang B (2011) The effect of inclusion waviness and waviness distribution on elastic properties of fiber-reinforced composites. Compos Part B Eng 42(1):62-70

69. Hsiao H, Daniel I (1996) Elastic properties of composites with fiber waviness. Compos Part A Appl Sci Manuf 27(10):931-941

70. Hu N, Fukunaga H, Lu C, Kameyama M, Yan B (2005) Prediction of elastic properties of carbon nanotube reinforced composites. Proc R Soc Lond A Math Phys Eng Sci 461(2058):1685-1710

71. Iijima S, Brabec C, Maiti A, Bernholc J (1996) Structural flexibility of carbon nanotubes. J Chem Phys 104(5):2089-2092

72. Jiang Q, Li Y, Xie J, Sun J, Hui D, Qiu Y (2013) Plasma functionalization of bucky paper and its composite with phenylethynyl-terminated polyimide. Compos Part B Eng 45(1):1275-1281

73. Kalamkarov A, Georgiades A, Rokkam S, Veedu V, Ghasemi-Nejhad M (2006) Analytical and numerical techniques to predict carbon nanotubes properties. Int J Solids Struct 43(22):6832-6854

74. Kalita K, Mukhopadhyay T, Dey P, Haldar S (2020) Genetic programming-assisted multi-scale optimization for multi-objective dynamic performance of laminated composites: the advantage of more elementary-level analyses. Neural Comput Appl 32(12):7969-7993

75. Kim YA, Muramatsu H, Hayashi T, Endo M, Terrones M, Dresselhaus MS (2006) Fabrication of high-purity, double-walled carbon nanotube buckypaper. Chem Vap Depos 12(6):327-330

76. Kirtania S, Chakraborty D (2007) Finite element based characterization of carbon nanotubes. J Reinf Plast Compos 26(15):1557-1570

77. Korobeynikov S, Alyokhin V, Babichev A (2018) On the molecular mechanics of single layer graphene sheets. Int J Eng Sci 133:109-131

78. Krenchel H (1964) Fibre reinforcement. Akademisk Forlag, Copenhagen

79. Krishnan A, Dujardin E, Ebbesen TW, Yianilos PN, Treacy MMJ (1998) Young's modulus of single-walled nanotubes. Phys Rev B 58:14013-14019

80. Kumar R, Mukhopadhyay T, Naskar S, Pandey K, Dey S (2019) Stochastic low-velocity impact analysis of sandwich plates including the effects of obliqueness and twist. Thin Walled Struct 145:106411
81. Kushvaha V, Kumar SA, Madhushri P (2019) Dynamic fracture toughness index: a new integrated methodology for mode-I fracture behaviour of polymer composite under impact loading. Mater Res Express 6(11):115342

82. Kushvaha V, Kumar SA, Madhushri P, Sharma A (2020) Artificial neural network technique to predict dynamic fracture of particulate composite. J Compos Mater 54(22):3099-3108

83. Lahiff E, Leahy R, Coleman JN, Blau WJ (2006) Physical properties of novel free-standing polymer-nanotube thin films. Carbon 44(8):1525-1529

84. Lambin P, Fonseca A, Vigneron J, Nagy J, Lucas A (1995) Structural and electronic properties of bent carbon nanotubes. Chem Phys Lett 245(1):85-89

85. Lee N, Chung D, Han I, Kang J, Choi Y, Kim H, Park S, Jin Y, Yi W, Yun M, Jung J, Lee C, You J, Jo S, Lee C, Kim J (2001) Application of carbon nanotubes to field emission displays. Diam Relat Mater 10(2):265-270. Proceedings of the 3rd specialist meeting on amorphous carbon

86. Lengvarský P, Bocko J (2015) Prediction of young's modulus of graphene sheets by the finite element method. Am J Mech Eng 3:225-229

87. Li C, Chou T-W (2003) Elastic moduli of multi-walled carbon nanotubes and the effect of van der Waals forces. Compos Sci Technol 63(11):1517-1524. Modeling and characterization of nanostructured materials

88. Li C, Chou T-W (2003) A structural mechanics approach for the analysis of carbon nanotubes. Int J Solids Struct 40(10):2487-2499

89. Li Z, Downes R, Liang Z (2015) In situ polymerized pCBT composites with aligned carbon nanotube buckypaper: structure and properties. Macromol Chem Phys 216(3):292-300

90. Li Z, Liang Z (2017) Optimization of buckypaper-enhanced multifunctional thermoplastic composites. Sci Rep 7:42423

91. Liu F, Song S, Xue D, Zhang H (2012) Folded structured graphene paper for high performance electrode materials. Adv Mater 24(8):1089-1094

92. Liu J, Zheng Q-S, Wang L-F, Jiang Q (2005) Mechanical properties of single-walled carbon nanotube bundles as bulk materials. J Mech Phys Solids 53(1):123-142

93. Liu L, Ma W, Zhang Z (2011) Macroscopic carbon nanotube assemblies: preparation, properties, and potential applications. Small 7(11):1504-1520

94. Liu T, Kumar S (2003) Effect of orientation on the modulus of SWNT films and fibers. Nano Lett 3:647-650

95. Liu T, Wang X (2007) Dynamic elastic modulus of single-walled carbon nanotubes in different thermal environments. Phys Lett A 365(1):144-148

96. Liu Y-N, Li M, Gu Y, Zhang Y, Li Q, Zhang Z (2015) Ultrastrong carbon nanotube/bismaleimide composite film with super-aligned and tightly packing structure. Compos Sci Technol 117:176-182

97. Loch AJK, Shaw SJ (1981) The fracture resistance of a toughened epoxy adhesive. J Adhes 12(1):59-77

98. López Polín G, Jaafar M, Guinea F, Roldán R, Gómez-Navarro C, Gómez-Herrero J (2015) Strain dependent elastic modulus of graphene. Carbon 124:42-48

99. Lu JP (1997) Elastic properties of carbon nanotubes and nanoropes. Phys Rev Lett 79:1297-1300

100. Lu X, Hu Z (2012) Mechanical property evaluation of singlewalled carbon nanotubes by finite element modeling. Compos Part B 43:1902-1913

101. Luo H, Lu G, Roy S, Lu H (2013) Characterization of the viscoelastic behavior of bismaleimide resin before and after exposure to high temperatures. Mech Time Depend Mater 17(3):369-399

102. Mahata A, Mukhopadhyay T (2018) Probing the chirality-dependent elastic properties and crack propagation 
behavior of single and bilayer stanene. Phys Chem Chem Phys 20:22768-22782

103. Mahmoudinezhad E, Ansari R, Basti A, Hemmatnezhad M (2012) An accurate spring-mass model for predicting mechanical properties of single-walled carbon nanotubes. Comput Mater Sci 62:6-11

104. Malakouti A, Montazeri A (2016) Nanomechanics analysis of perfect and defected graphene sheets via a novel atomic-scale finite element method. Superlattices Microstruct 94:1-12

105. Mawhinney DB, Naumenko V, Kuznetsova A, Yates JT, Liu J, Smalley R (2000) Surface defect site density on single walled carbon nanotubes by titration. Chem Phys Lett 324(1):213-216

106. Menon M, Srivastava D (1998) Carbon nanotube based molecular electronic devices. J Mater Res 13(9):2357-2362

107. Meo M, Rossi M (2006) Prediction of young's modulus of single wall carbon nanotubes by molecular-mechanics based finite element modelling. Compos Sci Technol 66(11):1597-1605

108. Miao M, Hawkins SC, Cai JY, Gengenbach TR, Knott R, Huynh CP (2011) Effect of gamma-irradiation on the mechanical properties of carbon nanotube yarns. Carbon 49(14):4940-4947

109. Miao M, McDonnell J, Vuckovic L, Hawkins SC (2010) Poisson's ratio and porosity of carbon nanotube dry-spun yarns. Carbon 48(10):2802-2811

110. Mohammadpour E, Awang M (2011) Predicting the nonlinear tensile behavior of carbon nanotubes using finite element simulation. Appl Phys A 104(2):609-614

111. Mohammadpour E, Awang M (2012) Nonlinear finite-element modeling of graphene and single and multi-walled carbon nanotubes under axial tension. Appl Phys A Mater Sci Process 106:581-588

112. Muc A (2010) Design and identification methods of effective mechanical properties for carbon nanotubes. Mater Des 31(4):1671-1675. Design of nanomaterials and nanostructures

113. Mukhopadhyay T, Mahata A, Adhikari S, Asle Zaeem M (2018) Probing the shear modulus of two-dimensional multiplanar nanostructures and heterostructures. Nanoscale 10:5280-5294

114. Mukhopadhyay T, Mahata A, Adhikari S, Zaeem MA (2017) Effective elastic properties of two dimensional multiplanar hexagonal nanostructures. 2D Mater 4(2):025006

115. Mukhopadhyay T, Mahata A, Adhikari S, Zaeem MA (2017) Effective mechanical properties of multilayer nano-heterostructures. Sci Rep 7(1):1-13

116. Mukhopadhyay T, Mahata A, Naskar S, Adhikari S (2020) Probing the effective young's modulus of 'magic angle' inspired multi-functional twisted nano-heterostructures. Adv Theory Simul 3(10):2000129

117. Mukhopadhyay T, Naskar S, Chakraborty S, Karsh P, Choudhury R, Dey S (2021) Stochastic oblique impact on composite laminates: a concise review and characterization of the essence of hybrid machine learning algorithms. Arch Comput Methods Eng 28(3):1731-1760

118. Nam TH, Goto K, Nakayama H, Oshima K, Premalal V, Shimamura Y, Inoue Y, Naito K, Kobayashi S (2014) Effects of stretching on mechanical properties of aligned multi-walled carbon nanotube/epoxy composites. Compos Part A Appl Sci Manuf 64:194-202

119. Naskar S, Mukhopadhyay T, Sriramula S (2019) Spatially varying fuzzy multi-scale uncertainty propagation in unidirectional fibre reinforced composites. Compos Struct 209:940-967

120. Nishijima H, Akita S, Nakayama Y (1999) Novel process for fabricating nanodevices consisting of carbon nanotubes. Jpn J Appl Phys 38(Part 1, No. 12B):7247-7252

121. Noh YJ, Kim HS, Kim SY (2014) Carbon nanotube mat reinforced thermoplastic composites with a polymerizable, low-viscosity cyclic butylene terephthalate matrix. Macromol Res 22(11):1183-1189

122. Nouri N, Ziaei-Rad S (2012) Mechanical property evaluation of buckypaper/epoxy composites using molecular dynamics simulations fully implemented on graphical processing units. J Comput Theor Nanosci 9(12):2144-2154

123. Novoselov KS, Geim AK, Morozov SV, Jiang D, Zhang Y, Dubonos SV, Grigorieva IV, Firsov AA (2004) Electric field effect in atomically thin carbon films. Science 306(5696):666-669

124. Ogasawara T, Moon S-Y, Inoue Y, Shimamura Y (2011) Mechanical properties of aligned multi-walled carbon nanotube/epoxy composites processed using a hot-melt prepreg method. Compos Sci Technol 71(16):1826-1833

125. Papanikos P, Nikolopoulos D, Tserpes K (2008) Equivalent beams for carbon nanotubes. Comput Mater Sci 43(2):345-352

126. Patole SP, Arif MF, Kumar S (2018) Polyvinyl alcohol incorporated buckypaper composites for improved multifunctional performance. Compos Sci Technol 168:429-436

127. Patole SP, Arif MF, Susantyoko RA, Almheiri S, Kumar S (2018) A wet-filtration-zipping approach for fabricating highly electroconductive and auxetic graphene/carbon nanotube hybrid buckypaper. Sci Rep 8(1):1-12

128. Pham GT, Park Y-B, Wang S, Liang Z, Wang B, Zhang C, Funchess P, Kramer L (2008) Mechanical and electrical properties of polycarbonate nanotube buckypaper composite sheets. Nanotechnology 19(32):325705

129. Quantum Composites. Advanced molding compound. Datasheets. Accessed 01 Sept 2021

130. Rafiee MA, Rafiee J, Wang Z, Song H, Yu Z-Z, Koratkar N (2009) Enhanced mechanical properties of nanocomposites at low graphene content. ACS Nano 3(12):3884-3890

131. Rafiee R, Eskandariyun A (2017) Comparative study on predicting young's modulus of graphene sheets using nano-scale continuum mechanics approach. Phys E Low Dimens Syst Nanostruct 90:42-48

132. Rafiee R, Ghorbanhosseini A (2017) Stochastic multi-scale modeling of randomly grown CNTs on carbon fiber. Mech Mater 106:1-7

133. Rafiee R, Heidarhaei M (2012) Investigation of chirality and diameter effects on the Young's modulus of carbon nanotubes using non-linear potentials. Compos Struct 94:2460-2464

134. Rahmandoust M, Öchsner A (2012) On finite element modeling of single- and multi-walled carbon nanotubes. J Nanosci Nanotechnol 12:8129-8136

135. Rangel J, Brostow W, Castano V (2013) Mechanical modeling of a single-walled carbon nanotube using the finite element approach. Polimery 58:276-281

136. Ranjbartoreh AR, Wang B, Shen X, Wang G (2011) Advanced mechanical properties of graphene paper. J Appl Phys 109(1):014306

137. Reddy CD, Rajendran S, Liew KM (2005) Equivalent continuum modeling of graphene sheets. Int J Nanosci 04(04):631-636

138. Rangapuram M K R (2019) Performance evaluation of BMI resin system for thin-ply composites. Masters Thesis, 7948

139. Rein M, Breuer O, Wagner H (2011) Sensors and sensitivity: carbon nanotube buckypaper films as strain sensing devices. Compos Sci Technol 71(3):373-381

140. Rinzler A, Liu J, Dai H, Nikolaev P, Huffman C, RodríguezMacías F, Boul P, Lu A, Heymann D, Colbert D, Lee R, Fischer J, Rao A, Eklund P, Smalley R (1998) Large-scale purification of single-wall carbon nanotubes: process, product, and characterization. Appl Phys A 67(1):29-37

141. Roy A, Gupta K, Naskar S, Mukhopadhyay T, Dey S (2021) Compound influence of topological defects and heteroatomic 
inclusions on the mechanical properties of SWCNTs. Mater Today Commun 26:102021

142. Sakhaee-Pour A (2009) Elastic properties of single-layered graphene sheet. Solid State Commun 149(1):91-95

143. Salvetat J-P, Briggs GAD, Bonard J-M, Bacsa RR, Kulik AJ, Stöckli T, Burnham NA, Forró L (1999) Elastic and shear moduli of single-walled carbon nanotube ropes. Phys Rev Lett 82:944-947

144. Savvas D, Papadopoulos V, Papadrakakis M (2012) The effect of interfacial shear strength on damping behavior of carbon nanotube reinforced composites. Int J Solids Struct 49(26):3823-3837

145. Scarpa F, Adhikari S, Chowdhury R (2010) The transverse elasticity of bilayer graphene. Phys Lett A 374(19):2053-2057

146. Scarpa F, Adhikari S, Phani AS (2009) Effective elastic mechanical properties of single layer graphene sheets. Nanotechnology 20(6):065709

147. Scarpa F, Adhikari S, Wang CY (2009) Mechanical properties of non-reconstructed defective single-wall carbon nanotubes. J Phys D Appl Phys 42(14):142002

148. Schiebold M, Mehner J (2018) A finite element approach for modeling of CNT-polymer-composites for use in large-area pressure sensors. 2018 Symposium on Design, Test, Integration and Packaging of MEMS and MOEMS (DTIP): $1-6$

149. Sharma A, Kushvaha V (2020) Predictive modelling of fracture behaviour in silica-filled polymer composite subjected to impact with varying loading rates using artificial neural network. Eng Fract Mech 239:107328

150. Sharma A, Mukhopadhyay T, Rangappa SM, Siengchin S, Kushvaha V (2021) Advances in computational intelligence of polymer composite materials: machine learning assisted modeling, analysis and design. https://doi.org/10.21203/rs.3.rs-471723/v1

151. Shi J, Natsuki T, Lei X, Ni Q (2014) Equivalent Young's modulus and thickness of graphene sheets for the continuum mechanical models. Appl Phys Lett 104:223101

152. Shokrieh MM, Rafiee R (2010) On the tensile behavior of an embedded carbon nanotube in polymer matrix with non-bonded interphase region. Compos Struct 92(3):647-652

153. Shokrieh MM, Rafiee R (2010) Prediction of mechanical properties of an embedded carbon nanotube in polymer matrix based on developing an equivalent long fiber. Mech Res Commun 37(2):235-240

154. Shokrieh MM, Rafiee R (2010) Prediction of Young's modulus of graphene sheets and carbon nanotubes using nanoscale continuum mechanics approach. Mater Des 31(2):790-795

155. Slobodian P, Riha P, Olejnik R, Matyas J, Kovar M (2018) Poisson effect enhances compression force sensing with oxidized carbon nanotube network/polyurethane sensor. Sens Actuators A Phys 271:76-82

156. Song L, Zhang H, Zhang Z, Xie S (2007) Processing and performance improvements of SWNT paper reinforced peek nanocomposites. Compos Part A Appl Sci Manuf 38(2):388-392

157. Suk JW, Piner RD, An J, Ruoff RS (2010) Mechanical properties of monolayer graphene oxide. ACS Nano 4(11):6557-6564

158. Sun X, Zhao W (2005) Prediction of stiffness and strength of single-walled carbon nanotubes by molecular-mechanics based finite element approach. Mater Sci Eng A 390(1):366-371

159. Susantyoko RA, Karam Z, Alkhoori S, Mustafa I, Wu C-H, Almheiri S (2017) A surface-engineered tape-casting fabrication technique toward the commercialisation of freestanding carbon nanotube sheets. J Mater Chem A 5:19255-19266

160. Swain A, Roy T, Nanda B (2013) Vibration behaviour of single walled carbon nanotube using finite element. Int J Theor Appl Res Mech Eng 2:130-133

161. Tanabi H, Erdal M (2019) Effect of CNTs dispersion on electrical, mechanical and strain sensing properties of CNT/epoxy nanocomposites. Results Phys 12:486-503
162. Tapia A, Peon-Escalante R, Villanueva C, Aviles F (2012) Influence of vacancies on the elastic properties of a graphene sheet. Comput Mater Sci 55:255-262

163. Terrones M, Banhart F, Grobert N, Charlier J-C, Terrones H, Ajayan PM (2002) Molecular junctions by joining single-walled carbon nanotubes. Phys Rev Lett 89:075505

164. Tsai C-H (2010) Elastic property prediction and variation quantification for buckypaper-polymer nanocomposites: modeling and experimental validation. $\mathrm{PhD}$ thesis

165. Tsai C-H, Zhang C, Jack DA, Wang B, Liang R (2011) Elastic property prediction of single-walled carbon nanotube buckypaper/polymer nanocomposites: stochastic bulk response modeling. J Nanosci Nanotechnol 11(3):2132-2141

166. Tserpes K (2012) Strength of graphenes containing randomly dispersed vacancies. Acta Mech 223:669-678

167. Tserpes K, Papanikos P (2005) Finite element modeling of single-walled carbon nanotubes. Compos Part B Eng 36(5):468-477

168. Tserpes K, Papanikos P, Labeas G, Pantelakis S (2008) Multiscale modeling of tensile behavior of carbon nanotube-reinforced composites. Theor Appl Fract Mech 49(1):51-60

169. Mukhopadhyay T, Kumar T, Dey S (2021) Probing the multiphysical probabilistic dynamics of a novel functional class of hybrid composite shells. Compos Struct 262:113294

170. Vigolo B, Pénicaud A, Coulon C, Sauder C, Pailler R, Journet C, Bernier P, Poulin P (2000) Macroscopic fibers and ribbons of oriented carbon nanotubes. Science 290(5495):1331-1334

171. Wang C, Peng Q, Wu J, He X, Tong L, Luo Q, Li J, Moody S, Liu H, Wang R, Du S, Li Y (2014) Mechanical characteristics of individual multi-layer graphene-oxide sheets under direct tensile loading. Carbon 80:279-289

172. Wang S, Downes R, Young C, Haldane D, Hao A, Liang R, Wang B, Zhang C, Maskell R (2015) Carbon fiber/carbon nanotube buckypaper interply hybrid composites: manufacturing process and tensile properties. Adv Eng Mater 17(10):1442-1453

173. Wernik JM, Meguid SA (2010) Atomistic-based continuum modeling of the nonlinear behavior of carbon nanotubes. Acta Mech 212(1):167-179

174. Wu H, Drzal LT (2012) Graphene nanoplatelet paper as a lightweight composite with excellent electrical and thermal conductivity and good gas barrier properties. Carbon 50(3):1135-1145

175. Wulfsberg J, Herrmann A, Ziegmann G, Lonsdorfer G, Stöß N, Fette M (2014) Combination of carbon fibre sheet moulding compound and prepreg compression moulding in aerospace industry. Procedia Eng 81:1601-1607. 11th International conference on technology of plasticity, ICTP 2014, 19-24 October 2014, Nagoya Congress Center, Nagoya, Japan

176. Xiao J, Staniszewski J, Gillespie J (2010) Tensile behaviors of graphene sheets and carbon nanotubes with multiple stone-wales defects. Mater Sci Eng A 527(3):715-723

177. Xu F, Wei B, Liu W, Zhu H, Zhang Y, Qiu Y (2015) In-plane mechanical properties of carbon nanotube films fabricated by floating catalyst chemical vapor decomposition. J Mater Sci 50(24):8166-8174

178. Yoon Y-G, Mazzoni MSC, Choi HJ, Ihm J, Louie SG (2001) Structural deformation and intertube conductance of crossed carbon nanotube junctions. Phys Rev Lett 86:688-691

179. Yu M-F, Files BS, Arepalli S, Ruoff RS (2000) Tensile loading of ropes of single wall carbon nanotubes and their mechanical properties. Phys Rev Lett 84:5552-5555

180. Zaeri M, Ziaei-Rad S, Vahedi A, Karimzadeh F (2010) Mechanical modelling of carbon nanomaterials from nanotubes to buckypaper. Carbon 48(13):3916-3930

181. Zhang J, Xu Y, Cui L, Fu A, Yang W, Barrow C, Liu J (2015) Mechanical properties of graphene films enhanced by homo-telechelic functionalized polymer fillers via $\pi-\pi$ stacking interactions. Compos Part A Appl Sci Manuf 71:1-8 
182. Zhang X, Jiang K, Feng C, Liu P, Zhang L, Kong J, Zhang T, Li Q, Fan S (2006) Spinning and processing continuous yarns from 4-inch wafer scale super-aligned carbon nanotube arrays. Adv Mater 18(12):1505-1510

183. Zhang Y, Chen X, Wang X (2008) Effects of temperature on mechanical properties of multi-walled carbon nanotubes. Compos Sci Technol 68(2):572-581

184. Zhao J, Jiang J-W, Wang L, Guo W, Rabczuk T (2014) Coarsegrained potentials of single-walled carbon nanotubes. J Mech Phys Solids 71:197-218

185. Zhao P, Shi G (2011) Study of Poisson's ratios of graphene and single-walled carbon nanotubes based on an improved molecular structural mechanics model. Comput Mater Contin 5(1):49-58

186. Zhou D, Seraphin S (1995) Complex branching phenomena in the growth of carbon nanotubes. Chem Phys Lett 238(4):286-289
187. Zhupanska OI (2013) The effect of orientational distribution of nanotubes on buckypaper nanocomposite mechanical properties. Mech Adv Mater Struct 20(1):1-10

188. Zuberi M, Esat V (2014) Estimating the effect of chirality and size on the mechanical properties of carbon nanotubes through finite element modelling. In: ASME 2014 12th biennial conference on engineering systems design and analysis, vol 1, pp 1-7

Publisher's Note Springer Nature remains neutral with regard to jurisdictional claims in published maps and institutional affiliations. 